\title{
A semi-discrete scheme for the stochastic Landau-Lifshitz equation
}

\author{
François Alouges - Anne de Bouard • \\ Antoine Hocquet
}

Received: 21 January 2014 / Published online: 28 May 2014

(C) Springer Science+Business Media New York 2014

\begin{abstract}
We propose a new convergent time semi-discrete scheme for the stochastic Landau-Lifshitz-Gilbert equation. The scheme is only linearly implicit and does not require the resolution of a nonlinear problem at each time step. Using a martingale approach, we prove the convergence in law of the scheme up to a subsequence.
\end{abstract}

Keywords Landau-Lifshitz equation - Numerical analysis - Stochastic partial differential equations

Mathematics Subject Classification $\quad 35 \mathrm{~K} 55 \cdot 60 \mathrm{H} 15 \cdot 65 \mathrm{Z} 05$

\section{Introduction}

Ferromagnetic materials possess a spontaneous magnetization $m$ which evolution is classically modelized, when thermal fluctuations are negligible, according to the socalled Landau-Lifshitz equation (see e.g. [1,2])

$$
\left\{\begin{array}{l}
\partial_{t} m=-\alpha m \times\left(m \times \mathrm{H}_{\mathrm{eff}}\right)+m \times \mathrm{H}_{\mathrm{eff}}, \quad \text { in }(0, T) \times D \\
\partial_{n} m(t, x)=0 \text { on }(0, T) \times \partial D \\
m(0, x):=m_{0}(x), \quad x \in D
\end{array}\right.
$$

F. Alouges $\cdot$ A. de Bouard $(\varangle) \cdot$ A. Hocquet

CMAP, CNRS and Ecole Polytechnique, Route de Saclay, 91128 Palaiseau Cedex, France e-mail: debouard@cmap.polytechnique.fr

F. Alouges

e-mail: francois.alouges@polytechnique.edu

A. Hocquet

e-mail: antoine.hocquet@polytechnique.edu 
In (1), $D \subseteq \mathbb{R}^{3}$ is the domain occupied by the sample, $\alpha>0$ is a damping parameter. The effective field $\mathrm{H}_{\mathrm{eff}}:=-\frac{\partial \mathcal{E}(m)}{\partial m}$, where $\mathcal{E}(m)$ is the Brown energy of $m$ (see [3] for more details), which governs the dynamics, contains several terms according to different physical phenomena : exchange, anisotropy, stray field, external field, magnetostriction, etc. Notice that (1) preserves the local magnitude of the magnetization, namely, assuming $m_{0}(x) \in \mathbb{S}^{2}:=\left\{x \in \mathbb{R}^{3},|x|=1\right\}$, we formally have

$$
m(t, x) \in \mathbb{S}^{2}, \quad \forall(t, x) \in[0, T] \times D .
$$

There is an abundant literature on the mathematical properties of (1). We refer the reader to [4-11], and references therein for the state-of-the-art of the analysis of (1).

In [1], thermal fluctuations are taken into account by adding to (1) a (stochastic) noise term $\xi(t, x)$. Following the presentation given in [12-15], and focusing on the case where only the exchange term is considered (i.e. $\mathrm{H}_{\mathrm{eff}}=\Delta m$ ) we modify (1) to

$$
\left\{\begin{array}{l}
\partial_{t} m=-\alpha m \times(m \times \Delta m)+m \times(\Delta m+\xi), \quad \text { in }(0, T) \times D, \\
\partial_{n} m(t, x)=0 \text { on }(0, T) \times \partial D, \\
m(0, x):=m_{0}(x) \quad \forall x \in D .
\end{array}\right.
$$

According to physicists, $\xi$ should be a Gaussian space-time white noise (see for instance, the review article [16] and references therein), that is uncorrelated in space. However, due to the lack of regularity of the space time white noise, Eq. (3) is not expected to possess a well defined solution in this case, and we therefore consider in this article a more regular noise in space. Namely, let $W$ be a cylindrical Wiener process that is given by the expression

$$
W(t)=\sum_{i \in \mathbb{N}} \beta_{i}(t) e_{i},
$$

where $\left(e_{i}\right)_{i \in \mathbb{N}}$ denotes a complete orthonormal system of $L^{2}(D)^{3}$, and $\left(\beta_{i}\right)_{i \in \mathbb{N}}$ stands for a sequence of real valued and independent brownian motions. Then, writing for each $i \in \mathbb{N}, G_{i}:=G e_{i}$, we set

$$
\xi(t, x)=G \dot{W}=\sum_{i \in \mathbb{N}} \dot{\beta}_{i}(t) G_{i}(x),
$$

where $G$ is a given Hilbert-Schmidt operator from the space $L^{2}(D)^{3}$ into the space $H^{2}(D)^{3}$.

As long as we have not specified the choice of the stochastic integral, (3) may have different meanings [17]. It is well-known (see for instance [18]) that in order to satisfy the geometrical constraint (2), the product with the noise term must be understood in the Stratonovich sense, which leads to the stochastic Landau-Lifshitz equation (SLL), where, for simplicity, we have set the parameter $\alpha$ to one :

$\mathrm{d} m=(-m \times(m \times \Delta m)+m \times \Delta m) \mathrm{d} t+m \times \circ(G \mathrm{~d} W)$, for $(t, x) \in[0, T] \times D$. 
Here we have denoted by "o d" the Stratonovich differential, and we will denote by "d" the Ito differential. We set the initial condition $m(0, x)=m_{0}(x)$, for any $x \in D$.

In order to work with a non-anticipative integral, we change (4) to its Ito form. Using the formal relation between the Stratonovich and Ito differentials, a corresponding Ito formulation of (4) is obtained by adding a correction term to the drift of (4). This term is what we may call in the sequel "the Ito correction". In this sense, the noise term can be rewritten as follows (see e.g. $[12,13,15])$ :

$$
m \times \circ(G \mathrm{~d} W)=m \times(G \mathrm{~d} W)+\frac{1}{2} \sum_{i \in \mathbb{N}}\left(m \times G_{i}\right) \times G_{i} \mathrm{~d} t .
$$

Noticing furthermore that $m \times(m \times \Delta m)$ formally equals $-\Delta m-m|\nabla m|^{2}$, we rewrite Eq. (4) as

$$
\mathrm{d} m=\left(\Delta m+m|\nabla m|^{2}+m \times \Delta m+\frac{1}{2} \sum_{i \in \mathbb{N}}\left(m \times G_{i}\right) \times G_{i}\right) \mathrm{d} t+m \times(G \mathrm{~d} W) .
$$

We refer to [12] for a review of the existing results on Eq. (5).

The so-called Gilbert form (SLLG) is (still formally) obtained by applying the operator $(\mathrm{Id}-m \times \cdot)$ to the previous equation :

$$
\begin{aligned}
\mathrm{d} m-m \times \mathrm{d} m= & {\left[2\left(\Delta m+m|\nabla m|^{2}\right)+\frac{1}{2} \sum_{i \in \mathbb{N}}(\mathrm{Id}-m \times)\left(\left(m \times G_{i}\right) \times G_{i}\right)\right] \mathrm{d} t } \\
& +(\mathrm{Id}-m \times)(m \times(G \mathrm{~d} W))
\end{aligned}
$$

Equivalence between (5) and (6) is not clearly stated in the litterature and we therefore establish it in Remark 2.

Developing numerical schemes for the simulation of LLG plays a prominent role in the modeling of ferromagnetic materials. We refer the reader to [19-21] for an overview of the literature on the subject. However, reliable schemes for the simulation of (SLL, SLLG) remain very few. Probably the first scheme for which convergence can be proved is given in [13] and is based on a Crank-Nicolson-type time-marching evolution which relies on a nonlinear iteration solved by a fixed point method. On the other hand, there has been in the past recent years an intensive development of a new class of numerical methods for LLG, based on a linear iteration, and for which unconditional convergence and stability can be shown [22-25]. The aim of this paper is to extend the ideas developed there and generalize the scheme in order to take into account the stochastic term. Note that this approach has already been used in [26] where a fully discrete scheme for (4) but with a one-dimensional noise is studied. The method used in that paper is based on the so-called Doss-Sussmann technique $[27,28]$, which, thanks to a geometric transformation, allows to replace the stochastic PDE by an equivalent PDE with random coefficients. However, it is well known that this method only works with a one-dimensional noise, and cannot be generalized to our setting. Instead, in the present paper, we apply the projection scheme directly on the original stochastic equation. This allows to consider a more general noise, but requires 
a specific treatment of the corresponding term. We think that the methodology that we develop can be generalized to stochastic differential or partial differential equations with a geometrical constraint. It is definitely different from-though related to- the approach of [29] (see Remark 1 below).

We only consider here a time semi-discrete approximation of (SLL, SLLG) for which we show the unconditional convergence when the time step tends to 0. Proving the convergence of the fully discrete approximation (using a finite element method in space) would not cause any major difficulty (see [23] for details).

Notation. Throughout this paper, we assume that $T>0$ is a given constant and $\left(\Omega, \mathcal{F}, \mathbb{P},\left(\mathcal{F}_{t}\right)_{t \in[0, T]},\left(W_{t}\right)_{t \in[0, T]}\right)$ is a stochastic basis, that is $(\Omega, \mathcal{F}, \mathbb{P})$ is a probability space, $\left(\mathcal{F}_{t}\right)_{t \in[0, T]}$ is a filtration and $\left(W_{t}\right)_{t \in[0, T]}$ a cylindrical Wiener process adapted to $\left(\mathcal{F}_{t}\right)$. The domain $D \subset \mathbb{R}^{3}$ is supposed to be bounded ; we denote by $a \cdot b$, where $a, b \in \mathbb{R}^{3}$ (resp. $\mathbb{R}^{3 \times 3}$ ), the standard scalar product in $\mathbb{R}^{3}$ (resp. $\mathbb{R}^{3 \times 3}$ ), and by $|\cdot|$ the associated euclidean norm. Norms in Banach spaces are in turn denoted by $\|\cdot\|$. In particular, the notation $\|f\|_{p, x}$ will be used to designate indifferently the $L^{p}(D)^{3}$ or $L^{p}(D)^{3 \times 3}$ norm. The inner product in the space $L^{2}(D)^{3}$ (respectively $L^{2}(D)^{3 \times 3}$ ) of square integrable functions with values in $\mathbb{R}^{3}$ (respectively $\mathbb{R}^{3 \times 3}$ ) is denoted by $(\cdot, \cdot)_{2, x}$, namely

$$
\forall f, g \in L^{2}(D)^{3}, \quad(f, g)_{2, x}:=\int_{D} f(x) \cdot g(x) \mathrm{d} x,
$$

and

$$
\forall F, G \in L^{2}(D)^{3 \times 3}, \quad(F, G)_{2, x}:=\int_{D} F(x) \cdot G(x) \mathrm{d} x .
$$

The notation $\mathcal{C}([0, T] ; X)$, where $X$ is a Banach space, is used to denote the space of continuous functions from $[0, T]$ into $X$. Classical Sobolev spaces of $\mathbb{R}^{3}$-valued functions are denoted by $W^{\alpha, p}(D), \alpha \in \mathbb{R}$, or $H^{\alpha}(D)$ when $p=2$, (see e.g. [30]). Finally, the norm of a Hilbert-Schmidt operator from $L^{2}(D)$ into $H^{\alpha}(D)$ is denoted by $\|\cdot\|_{2, \alpha}$. For a given number of time intervals $N \in \mathbb{N}^{*}$, we define the time step $\Delta t:=\frac{T}{N}$, and $\Delta W_{N}^{n}:=W((n+1) \Delta t)-W(n \Delta t)$, for any $n$ with $0 \leq n \leq N-1$. Therefore $G \Delta W_{N}^{n}$ is a gaussian random variable on $L^{2}(D)^{3}$ with covariance operator $(\Delta t) G G^{*}$.

\section{Main result}

Our purpose is to analyse a semi-implicit scheme with parameter $\theta \in\left(\frac{1}{2}, 1\right]$. Unlike the approach used in [13], we use the Gilbert form of the equation, i.e. Eq. (6). This approach allows us to overcome the difficulty of solving a nonlinear system at each step of the algorithm. Given the data $m_{N}^{n}$, where $N$ is the number of time steps, and $n \in\{0, \ldots, N-1\}$, which is an approximation of $m(n \Delta t)$, the unknown $v_{N}^{n}$, namely the tangential increment of $m_{N}^{n}$, can be found simply by solving a linear system. Indeed, following an idea of [23], one may search $v_{N}^{n}$ in the subset of $H^{1}(D)^{3}$ 
whose elements are almost everywhere orthogonal to $m_{N}^{n}$, so that the non linear term in $m_{N}^{n} \times\left(m_{N}^{n} \times \Delta m_{N}^{n}\right)=-\Delta m_{N}^{n}-m_{N}^{n}\left|\nabla m_{N}^{n}\right|^{2}$ vanishes when testing against functions that also satisfy this constraint. Roughly speaking, the test functions in the following formulation (10) "only see" the part of $m_{N}^{n+1}-m_{N}^{n}$ which is orthogonal to $m_{N}^{n}$, but this is however sufficient, as shown in Sect. 6.

Let us now describe the scheme rigorously. We fix the parameter

$$
\theta \in\left(\frac{1}{2}, 1\right]
$$

and assume that the operator $G: L^{2}(D)^{3} \rightarrow H^{2}(D)^{3}$ satisfies

$$
\|G\|_{2,2}^{2}=\sum_{i \in \mathbb{N}}\left\|G_{i}\right\|_{H^{2}(D)^{3}}^{2}<\infty .
$$

Our algorithm reads as follows, for a given integer $N>0$ :

Algorithm (*) : Fix

$$
m_{N}^{0}:=m_{0} \in H^{1}(D)^{3},
$$

and for any $n \in\{0, \ldots, N-1\}$, suppose that the random variable $m_{N}^{n}(\omega, \cdot) \in H^{1}(D)^{3}$ is known. Let $v_{N}^{n}(\omega, \cdot)$ be the unique solution in the space

$$
\mathbb{W}_{N, n}(\omega):=\left\{\psi \in H^{1}(D)^{3}, \forall x \in D, \psi(x) \perp m_{N}^{n}(\omega, x)\right\}
$$

of the variational problem $: \forall \varphi \in \mathbb{W}_{N, n}(\omega)$,

$$
\begin{aligned}
& \left(v_{N}^{n}-m_{N}^{n} \times v_{N}^{n}, \varphi\right)_{2, x}+2 \theta \Delta t\left(\nabla v_{N}^{n}, \nabla \varphi\right)_{2, x} \\
& =-2 \Delta t\left(\nabla m_{N}^{n}, \nabla \varphi\right)_{2, x}+\left(\left(\operatorname{Id}-m_{N}^{n} \times\right)\left(m_{N}^{n} \times G \Delta W_{N}^{n}\right), \varphi\right)_{2, x} \\
& \quad+\frac{\Delta t}{2} \sum_{i \in \mathbb{N}}\left(\left(\operatorname{Id}-m_{N}^{n} \times\right)\left(\left(m_{N}^{n} \times G_{i}\right) \times G_{i}\right), \varphi\right)_{2, x} .
\end{aligned}
$$

Then, we set, for all $(\omega, x) \in \Omega \times D$,

$$
m_{N}^{n+1}(\omega, x)=\frac{m_{N}^{n}(\omega, x)+v_{N}^{n}(\omega, x)}{\left|m_{N}^{n}(\omega, x)+v_{N}^{n}(\omega, x)\right|} .
$$

Note that the formulation (10) is a $\theta$-scheme applied to the variational formulation of Eq. (6) (see [23]). One has $m_{N}^{n} \in H^{1}(D)^{3}$ a.s., for any $n \in\{0, \ldots, N\}$ and $\left(m_{N}^{n}\right)_{0 \leq n \leq N}$ is adapted to the filtration $\left(\mathbb{F}_{N}^{n}\right)_{0 \leq n \leq N}$ defined by

$$
\mathbb{F}_{N}^{n}:=\sigma\{G W(k \Delta t), 0 \leq k \leq n\}
$$

Indeed, it is not difficult to prove that under the above assumptions, problem (10) admits a unique solution $v_{N}^{n}(\omega, \cdot) \in \mathbb{W}_{N, n}(\omega)$ (see [23] for a proof in the deter- 
ministic case). The noise and correction terms do not alter the hypotheses of the Lax-Milgram theorem. Moreover, this solution depends continuously in $H^{1}(D)^{3}$ on the two arguments $\left(m_{N}^{n}, G \Delta W_{N}^{n}\right)$, for the $H^{1}(D)^{3} \times L^{2}(D)^{3}$ topology. It implies in particular that the law of $v_{N}^{n}$ on $H^{1}(D)^{3}$ only depends on the law of $\left(m_{N}^{n}, G \Delta W_{N}^{n}\right)$ on $H^{1}(D)^{3} \times L^{2}(D)^{3}$.

Remark 1 As mentioned before, the approach here is different from the one in [29], where the approximation of solutions of some Stratonovich stochastic differential equation with values in a manifold is considered. Indeed, in [29], the scheme consists in using the explicit Euler scheme (which approximates the Ito equation) on one time step, and then projecting the solution on the manifold. Here, we do not approximate the Itô equation, since part of the Itô correction is put in the increment. We will see that the projection on the manifold (the sphere here) brings the remaining part of the Itô correction.

We now give the definition of the martingale solutions of Eq. (5) that we consider here, which is similar to the one in $[13,15])$.

Definition 1 (Martingale solution) Given $T>0$, a martingale solution on $[0, T]$ of (5) is given by a filtered probability space $\left(\tilde{\Omega}, \tilde{\mathcal{F}}, \tilde{\mathbb{P}},\left(\tilde{\mathcal{F}}_{t}\right)\right)$, together with $H^{1}(D)^{3}$ valued, progressively measurable processes $G \tilde{W}$ and $\tilde{m}$ defined on this space, where $G \tilde{W}$ is a Wiener process on $\left(\tilde{\Omega}, \tilde{\mathcal{F}}, \tilde{\mathbb{P}},\left(\tilde{\mathcal{F}}_{t}\right)\right)$, with covariance operator $G G^{*}$, and $\tilde{m}$ satisfies the following assumptions :

1. $\tilde{m}(\omega, \cdot) \in C\left([0, T] ; L^{2}(D)^{3}\right)$, a.s.

2. for any $t \in[0, T], \tilde{m}(t)$ belongs to $H^{1}(D)^{3}$, and the random variable $\Delta \tilde{m}+$ $\tilde{m}|\nabla \tilde{m}|^{2}+\tilde{m} \times \Delta \tilde{m}+\frac{1}{2} \sum_{i \in \mathbb{N}}\left(\tilde{m} \times G_{i}\right) \times G_{i}$ takes its values in the space $L^{1}\left(0, T ; L^{2}(D)^{3}\right)$, a.s.

3. $\tilde{m}$ satisfies (5); more precisely,

$$
\begin{aligned}
\tilde{m}(t)= & m_{0}+\int_{0}^{t}\left(\Delta \tilde{m}(s)+\tilde{m}(s)|\nabla \tilde{m}(s)|^{2}+\tilde{m}(s) \times \Delta \tilde{m}(s)\right. \\
& \left.+\frac{1}{2} \sum_{i \in \mathbb{N}}\left(\tilde{m}(s) \times G_{i}\right) \times G_{i}\right) \mathrm{d} s+\int_{0}^{t} \tilde{m}(s) \times(G \mathrm{~d} \tilde{W}(s))
\end{aligned}
$$

where the first integral is the Bochner integral in $L^{2}(D)^{3}$, and the second is the Ito integral of a predictable $H^{1}(D)^{3}$-valued process.

4. $|\tilde{m}(\omega, t, x)|=1$ for almost every $(\omega, t, x) \in \tilde{\Omega} \times[0, T] \times D$.

Remark 2 Note that if $\tilde{m}$ is a martingale solution of (5), then we can rewrite the stochastic integral of the predictable process $s \mapsto \tilde{m}(s) \times$ with respect to the semimartingale $\tilde{m}$ as 


$$
\begin{aligned}
& \int_{0}^{t} \tilde{m}(s) \times \mathrm{d} \tilde{m}(s) \\
& \quad=\int_{0}^{t} \tilde{m}(s) \times \mathrm{d}\left(\int_{0}^{s} I(\sigma) \mathrm{d} \sigma\right)+\int_{0}^{t} \tilde{m}(s) \times \mathrm{d}\left(\int_{0}^{s} \tilde{m}(\sigma) \times G \mathrm{~d} \tilde{W}(\sigma)\right)
\end{aligned}
$$

where $I$ is given by $\forall s \in[0, T]$ :

$$
\begin{aligned}
I(s)= & \Delta \tilde{m}(s)+\tilde{m}(s)|\nabla \tilde{m}(s)|^{2}+\tilde{m}(s) \times \Delta \tilde{m}(s) \\
& +\frac{1}{2} \sum_{i \in \mathbb{N}}\left(\tilde{m}(s) \times G_{i}\right) \times G_{i} \in L^{2}(\Omega \times D)^{3} .
\end{aligned}
$$

It then follows from classical properties of stochastic integrals with respect to semimartingales that

$$
\int_{0}^{t}(\operatorname{Id}-\tilde{m}(s) \times) \mathrm{d} \tilde{m}(s)=\int_{0}^{t}(\operatorname{Id}-\tilde{m}(s) \times) I(s) \mathrm{d} s+\int_{0}^{t}(\operatorname{Id}-\tilde{m}(s) \times) G \mathrm{~d} \tilde{W}(\sigma),
$$

and since for almost all $\omega, t, x,|\tilde{m}(\omega, t, x)|=1, \tilde{m}$ is also a solution to (6). Thus (5) and (6) are in fact equivalent.

Our main result is then given by the following theorem, and says that, up to a subsequence, the discrete solution $m_{N}$ of the algorithm $(*)$ converges in law to a martingale solution of equation (5).

Theorem 1 (Convergence of the algorithm) For every $N \in \mathbb{N}^{*}$, we define the progressively measurable $H^{1}(D)^{3}$-valued process $m_{N}$ by:

$$
m_{N}(t):=m_{N}^{n} \text { if } t \in[n \Delta t,(n+1) \Delta t)
$$

There exists a martingale solution of $(5)\left(\tilde{\Omega}, \tilde{\mathcal{F}}, \tilde{\mathbb{P}},\left(\tilde{\mathcal{F}}_{t}\right)_{t \in[0, T]},\left(\tilde{W}_{t}\right)_{t \in[0, T]}, \tilde{m}\right)$, and a sequence $\left(\tilde{m}_{N}\right)_{N \in \mathbb{N} *}$ of random processes defined on $\tilde{\Omega}$, with the same law as $m_{N}$, so that up to a subsequence, the following convergence holds :

$$
\tilde{m}_{N} \underset{N \rightarrow \infty}{\longrightarrow} \tilde{m}, \quad \text { in } L^{2}(\tilde{\Omega} \times[0, T] \times D)^{3} .
$$

In order to prove Theorem 1, we proceed in several steps. In Sect. 3, we establish uniform estimates for several processes. We first establish a uniform bound on the Dirichlet energy of $m_{N}$, thanks to the variational formulation, and an appropriate choice of the test function. Section 4 is devoted to the proof of the tightness of the sequence $\left(m_{N}\right)$ on the space $L^{2}([0, T] \times D)^{3}$. After a change of probability space, we can assume that there exists an almost sure limit $m$ of $\left(m_{N}\right)$, that is

$$
m_{N} \underset{N \rightarrow \infty}{\longrightarrow} m \text { a.s. in } L^{2}([0, T] \times D)^{3} .
$$


Then, setting

$$
m_{N}(t)=m_{0}+F_{N}(t)+X_{N}(t)
$$

where $\left(X_{N}(n \Delta t)\right)_{0 \leq n \leq N-1}$ defines an $L^{2}(D)^{3}$-valued discrete parameter martingale, with respect to the filtration $\left(\mathbb{F}_{N}^{n}\right)_{0 \leq n \leq N-1}$ [see (12)], and $F_{N}(t)$ is, for each $N$, a deterministic function of $\left.m_{N}\right|_{[0, t]}$, we use (10) and the previous energy estimates to identify $F_{N}(t)$ and its limit up to a subsequence. In Sect. 5, we show that, still up to a subsequence, $X_{N}(t)$ converges to a limit $X(t)$ which is a square-integrable continuous martingale with an explicit quadratic variation. The martingale representation theorem allows us to conclude : there exists a new filtered probability space for which the limit of the martingale part is a stochastic integral with respect to a Wiener process $G \tilde{W}$ with covariance operator $G G^{*}$. Finally we use the limit of (13) and this latter stochastic integral in order to identify the equation satisfied by $m(t)$. The explicit form of the limit $F(t)$ of $F_{N}(t)$ as $N \rightarrow \infty$ is the Bochner integral of the $L^{2}(D)^{3}$-valued process $t^{\prime} \mapsto \Delta m\left(t^{\prime}\right)+m\left(t^{\prime}\right)\left|\nabla m\left(t^{\prime}\right)\right|^{2}+m\left(t^{\prime}\right) \times \Delta m\left(t^{\prime}\right)+\frac{1}{2} \sum_{i \in \mathbb{N}}\left(m\left(t^{\prime}\right) \times G_{i}\right) \times G_{i}$ on the time interval $[0, t]$, which allows us to conclude.

\section{Energy estimates}

Fix $N>0$, and set $m_{N}^{0}=m_{0}$. Let $\left(m_{N}^{n}\right)_{0 \leq n \leq N}$ and $\left(v_{N}^{n}\right)_{0 \leq n \leq N}$ be given by the algorithm (*). In all what follows, we write

$$
A_{N}^{n}:=m_{N}^{n} \times\left(G \Delta W_{N}^{n}\right)
$$

This term corresponds to the noise term which is added at each step of the algorithm. Thanks to the Gaussian properties of $G \Delta W_{N}^{n}$, the fact that $\left\|m_{N}^{n}\right\|_{L^{\infty}(D)^{3}} \leq 1$, and the Sobolev embeddings, we have the following obvious, but useful estimates : for all $n \in\{0, \ldots, N\}$,

$$
\mathbb{E}\left[\left\|A_{N}^{n}\right\|_{2, x}^{2}\right] \leq \Delta t\|G\|_{2,0}^{2}
$$

and

$$
\mathbb{E}\left[\left\|A_{N}^{n}\right\|_{L^{4}}^{4}\right] \leq C(\Delta t)^{2}\|G\|_{2,1}^{4}
$$

Proposition 1 There exists a constant $C=C\left(T, m_{0},\|G\|_{2,2}\right)$, so that for all $N \in \mathbb{N}^{*}$,

$$
\begin{aligned}
& \max _{n=0 \ldots N} \mathbb{E}\left[\left\|\nabla m_{N}^{n}\right\|_{2, x}^{2}\right] \leq C, \\
& \mathbb{E}\left[\sum_{n=0}^{N-1}\left\|v_{N}^{n}-A_{N}^{n}\right\|_{2, x}^{2}\right] \leq C \Delta t, \\
& \mathbb{E}\left[\sum_{n=0}^{N-1}\left\|v_{N}^{n}\right\|_{2, x}^{2}\right] \leq C, \\
& \mathbb{E}\left[\sum_{n=0}^{N-1}\left\|\nabla v_{N}^{n}\right\|_{2, x}^{2}\right] \leq C .
\end{aligned}
$$


The proof of Proposition 1 uses the following remark, together with the estimate of Lemma 1 below, whose proof is postponed to the end of Sect. 3.

Remark 3 The renormalization stage decreases the Dirichlet energy. Indeed, it was shown in [31] that for any map $\psi \in H^{1}(D)^{3}$, such that a.e. in $D,|\psi(x)| \geq 1$, one has

$$
\int_{D}\left|\nabla\left(\frac{\psi(x)}{|\psi(x)|}\right)\right|^{2} \mathrm{~d} x \leq \int_{D}|\nabla(\psi(x))|^{2} \mathrm{~d} x .
$$

Lemma 1 For all $\epsilon$ with $0<\epsilon<2 \theta-1$, there exists $C=C\left(\epsilon,\|G\|_{2,2}, T\right)>0$ such that for all $N \in \mathbb{N}^{*}$, and $n=0, \ldots, N-1$ :

$$
\begin{aligned}
& \mathbb{E}\left[\left\|\nabla m_{N}^{n+1}\right\|_{2, x}^{2}\right]+\frac{(1-\epsilon)}{\Delta t} \mathbb{E}\left[\left\|v_{N}^{n}-A_{N}^{n}\right\|_{2, x}^{2}\right]+(2 \theta-1-\epsilon) \mathbb{E}\left[\left\|\nabla v_{N}^{n}\right\|_{2, x}^{2}\right] \\
& \leq(1+C \Delta t) \mathbb{E}\left[\left\|\nabla m_{N}^{n}\right\|_{2, x}^{2}\right]+C \Delta t
\end{aligned}
$$

Let us now prove Proposition 1 with the help of Lemma 1.

Proof (Proposition 1) In the sequel, we fix $\epsilon \in(0,2 \theta-1)$. We first prove (17). We deduce from (22) that for all $n=0 \ldots N-1$

$$
\mathbb{E}\left[\left\|\nabla m_{N}^{n+1}\right\|_{2, x}^{2}\right] \leq(1+C \Delta t) \mathbb{E}\left[\left\|\nabla m_{N}^{n}\right\|_{2, x}^{2}\right]+C \Delta t .
$$

We then apply the discrete Gronwall lemma. There exists $C=C\left(\|G\|_{2,2}, T\right)>0$ such that for all $n=0 \ldots N$,

$$
\mathbb{E}\left[\left\|\nabla m_{N}^{n}\right\|_{2, x}^{2}\right] \leq C\left(1+\mathbb{E}\left[\left\|\nabla m_{0}\right\|_{2, x}^{2}\right]\right),
$$

and (17) is proved.

We now turn to the proof of (18)-(20). We note that (22) implies in particular

$$
\begin{aligned}
& \mathbb{E}\left[\left\|\nabla m_{N}^{n+1}\right\|_{2, x}^{2}\right]-\mathbb{E}\left[\left\|\nabla m_{N}^{n}\right\|_{2, x}^{2}\right]+\frac{(1-\epsilon)}{\Delta t} \mathbb{E}\left[\left\|v_{N}^{n}-A_{N}^{n}\right\|_{2, x}^{2}\right] \\
& +(2 \theta-1-\epsilon) \mathbb{E}\left[\left\|\nabla v_{N}^{n}\right\|_{2, x}^{2}\right] \leq C \Delta t \mathbb{E}\left[\left\|\nabla m_{N}^{n}\right\|_{2, x}^{2}\right]+C \Delta t .
\end{aligned}
$$

By summing these inequalities for $n=0 \ldots N-1$, we obtain

$$
\begin{aligned}
\mathbb{E}\left[\left\|\nabla m_{N}^{N}\right\|_{2, x}^{2}\right]-\mathbb{E}\left[\left\|\nabla m_{N}^{0}\right\|_{2, x}^{2}\right]+\frac{(1-\epsilon)}{\Delta t} \sum_{n=0}^{N-1} \mathbb{E}\left[\left\|v_{N}^{n}-A_{N}^{n}\right\|_{2, x}^{2}\right] \\
\quad+(2 \theta-1-\epsilon) \sum_{n=0}^{N-1} \mathbb{E}\left[\left\|\nabla v_{N}^{n}\right\|_{2, x}^{2}\right] \\
\leq \sum_{n=0}^{N-1} C \Delta t \mathbb{E}\left[\left\|\nabla m_{N}^{n}\right\|_{2, x}^{2}\right]+\sum_{n=0}^{N-1} C \Delta t \leq C\left(\|G\|_{2,2}, T, m_{0}\right),
\end{aligned}
$$


thanks to (17). This implies that :

$$
\begin{aligned}
& \frac{(1-\epsilon)}{\Delta t} \sum_{n=0}^{N-1} \mathbb{E}\left[\left\|v_{N}^{n}-A_{N}^{n}\right\|_{2, x}^{2}\right]+(2 \theta-1-\epsilon) \sum_{n=0}^{N-1} \mathbb{E}\left[\left\|\nabla v_{N}^{n}\right\|_{2, x}^{2}\right] \\
& \quad \leq C\left(\|G\|_{2,2}, T, m_{0}\right)-\mathbb{E}\left[\left\|\nabla m_{N}^{N}\right\|_{2, x}^{2}\right]+\mathbb{E}\left[\left\|\nabla m_{N}^{0}\right\|_{2, x}^{2}\right] \\
& \quad \leq C^{\prime}\left(\|G\|_{2,2}, T, m_{0}\right) .
\end{aligned}
$$

Thus, (18) and (20) follow. Finally, we may deduce (19) from (18) and (15). This ends the proof of Proposition 1.

We now turn to the proof of the lemma.

Proof (Lemma 1.) Let $0 \leq n \leq N-1$. Since, by definition of the variational problem (10), $m_{N}^{n}(x) \cdot v_{N}^{n}(x)=0$, almost everywhere, and almost surely, it follows that for a.e. $x \in D$, a.s.

$$
\left|m_{N}^{n}(x)+v_{N}^{n}(x)\right|=\sqrt{1+\left|v_{N}^{n}(x)\right|^{2}} \geq 1
$$

and thanks to Remark 3, one has a.s.

$$
\begin{aligned}
\left\|\nabla m_{N}^{n+1}\right\|_{2, x}^{2} & =\int_{D}\left|\nabla\left(\frac{m_{N}^{n}+v_{N}^{n}}{\left|m_{N}^{n}+v_{N}^{n}\right|}\right)\right|^{2} \mathrm{~d} x \\
& \leq \int_{D}\left|\nabla m_{N}^{n}+\nabla v_{N}^{n}\right|^{2} \mathrm{~d} x .
\end{aligned}
$$

Then, by expanding the right hand side of this inequality:

$$
\left\|\nabla m_{N}^{n+1}\right\|_{2, x}^{2} \leq\left\|\nabla m_{N}^{n}\right\|_{2, x}^{2}+2\left(\nabla m_{N}^{n}, \nabla v_{N}^{n}\right)_{2, x}+\left\|\nabla v_{N}^{n}\right\|_{2, x}^{2} .
$$

To find an expression of $2\left(\nabla m_{N}^{n}, \nabla v_{N}^{n}\right)_{2, x}$, we use (10) with the test function

$$
\varphi:=v_{N}^{n}-A_{N}^{n} \in \mathbb{W}_{N, n} .
$$

Then, observing that for any $x \in D$

$$
\begin{aligned}
& \left(v_{N}^{n}-m_{N}^{n} \times v_{N}^{n}\right)(x) \cdot\left(v_{N}^{n}-A_{N}^{n}\right)(x) \\
& \quad-\left(\operatorname{Id}-m_{N}^{n} \times\right)\left(m_{N}^{n} \times G \Delta W_{N}^{n}\right)(x) \cdot\left(v_{N}^{n}-A_{N}^{n}\right)(x) \\
& \quad=\left(\left(\operatorname{Id}-m_{N}^{n} \times\right)\left(v_{N}^{n}-A_{N}^{n}\right)(x)\right) \cdot\left(v_{N}^{n}(x)-A_{N}^{n}(x)\right) \\
& \quad=\left|v_{N}^{n}(x)-A_{N}^{n}(x)\right|^{2},
\end{aligned}
$$


one has

$$
\begin{aligned}
2\left(\nabla m_{N}^{n}, \nabla v_{N}^{n}\right)_{2, x}= & -\frac{1}{\Delta t}\left\|v_{N}^{n}-A_{N}^{n}\right\|_{2, x}^{2}-2 \theta\left\|\nabla v_{N}^{n}\right\|_{2, x}^{2} \\
& +2 \theta\left(\nabla v_{N}^{n}, \nabla A_{N}^{n}\right)_{2, x}+2\left(\nabla m_{N}^{n}, \nabla A_{N}^{n}\right)_{2, x} \\
& +\frac{1}{2} \sum_{i \in \mathbb{N}}\left(\left(\operatorname{Id}-m_{N}^{n} \times\right)\left(\left(m_{N}^{n} \times G_{i}\right) \times G_{i}\right), v_{N}^{n}-A_{N}^{n}\right)_{2, x},
\end{aligned}
$$

which, using (23) and taking the expectation yields to

$$
\begin{aligned}
\mathbb{E} & {\left[\left\|\nabla m_{N}^{n+1}\right\|_{2, x}^{2}\right]+\frac{1}{\Delta t} \mathbb{E}\left[\left\|v_{N}^{n}-A_{N}^{n}\right\|_{2, x}^{2}\right]+(2 \theta-1) \mathbb{E}\left[\left\|\nabla v_{N}^{n}\right\|_{2, x}^{2}\right] } \\
\leq & \mathbb{E}\left[\left\|\nabla m_{N}^{n}\right\|_{2, x}^{2}\right]+2 \theta \mathbb{E}\left[\left(\nabla v_{N}^{n}, \nabla A_{N}^{n}\right)_{2, x}\right]+2 \mathbb{E}\left[\left(\nabla m_{N}^{n}, \nabla A_{N}^{n}\right)_{2, x}\right] \\
& +\frac{1}{2} \mathbb{E}\left[\sum_{i \in \mathbb{N}}\left(\left(\mathrm{Id}-m_{N}^{n} \times\right)\left(\left(m_{N}^{n} \times G_{i}\right) \times G_{i}\right), v_{N}^{n}-A_{N}^{n}\right)_{2, x}\right],
\end{aligned}
$$

all terms on the left hand side being non negative, due to $\theta \in\left(\frac{1}{2}, 1\right]$.

Now, since $m_{N}^{n}$ and $G \Delta W_{N}^{n}$ are independent, and $\mathbb{E}\left[G \Delta W_{N}^{n}\right]=0$, we have

$$
\mathbb{E}\left[\left(\nabla m_{N}^{n}, \nabla A_{N}^{n}\right)_{2, x}\right]=0
$$

Moreover, by (14) and the Sobolev embedding $H^{2}(D)^{3} \subset L^{\infty}(D)^{3}$,

$$
\begin{aligned}
\mathbb{E}\left[\left\|\nabla A_{N}^{n}\right\|_{2, x}^{2}\right] & \leq 2\left(\mathbb{E}\left\|\nabla m_{N}^{n}\right\|_{2, x}^{2} \mathbb{E}\left[\left\|G \Delta W_{N}^{n}\right\|_{\infty, x}^{2}\right]+\mathbb{E}\left[\left\|\nabla\left(G \Delta W_{N}^{n}\right)\right\|_{2, x}^{2}\right]\right) \\
& \leq C \Delta t\|G\|_{2,2}^{2}\left(\mathbb{E}\left[\left\|\nabla m_{N}^{n}\right\|_{2, x}^{2}\right]+1\right) .
\end{aligned}
$$

Therefore

$$
\mathbb{E}\left[\left(\nabla v_{N}^{n}, \nabla A_{N}^{n}\right)_{2, x}\right] \leq \frac{\epsilon}{2} \mathbb{E}\left[\left\|\nabla v_{N}^{n}\right\|_{2, x}^{2}\right]+\frac{C \Delta t}{2 \epsilon}\|G\|_{2,2}^{2}\left(\mathbb{E}\left[\left\|\nabla m_{N}^{n}\right\|_{2, x}^{2}\right]+1\right)
$$

Similarly, one has

$$
\begin{aligned}
& \left.\mathbb{E}\left[\left(\sum_{i \in \mathbb{N}}\left(\operatorname{Id}-m_{N}^{n}\right) \times\left(\left(m_{N}^{n} \times G_{i}\right) \times G_{i}\right)\right), v_{N}^{n}-A_{N}^{n}\right)_{2, x}\right] \\
& \leq \frac{\Delta t}{2 \epsilon}\|G\|_{2,2}^{4}+\frac{2 \epsilon}{\Delta t} \mathbb{E}\left[\left\|v_{N}^{n}-A_{N}^{n}\right\|_{2, x}^{2}\right] .
\end{aligned}
$$


Using (26), (28) and (29) in (25) gives

$$
\begin{gathered}
\mathbb{E}\left[\left\|\nabla m_{N}^{n+1}\right\|_{2, x}^{2}\right]+\frac{1-\epsilon}{\Delta t} \mathbb{E}\left[\left\|v_{N}^{n}-A_{N}^{n}\right\|_{2, x}^{2}\right]+(2 \theta-1-\theta \epsilon) \mathbb{E}\left[\left\|\nabla v_{N}^{n}\right\|_{2, x}^{2}\right] \\
\leq \mathbb{E}\left[\left\|\nabla m_{N}^{n}\right\|_{2, x}^{2}\right]+\frac{C \theta \Delta t}{\epsilon}\|G\|_{2,2}^{2}\left(\mathbb{E}\left[\left\|\nabla m_{N}^{n}\right\|_{2, x}^{2}\right]+1\right)+\frac{\Delta t}{4 \epsilon}\|G\|_{2,2}^{4} .
\end{gathered}
$$

Since $\theta \leq 1$, this proves the lemma.

Let us fix $N \in \mathbb{N}^{*}$. Let $w_{N}^{n}=\frac{1}{\Delta t}\left(v_{N}^{n}-A_{N}^{n}\right)$ for $n=0, \ldots, N-1$. In the following, we will also denote by $v_{N}, w_{N}$, the piecewise constant processes (indexed by the time interval $[0, T]$ ), whose values on $[n \Delta t,(n+1) \Delta t)$ are (respectively) $v_{N}^{n}, w_{N}^{n}$. The previous energy estimates can now be written in the form:

$$
\begin{aligned}
& \underset{t \in[0, T]}{\operatorname{esssup}} \mathbb{E}\left[\left\|\nabla m_{N}(t, \cdot)\right\|_{2, x}^{2}\right] \leq C, \\
& \mathbb{E}\left[\int_{0}^{T}\left\|w_{N}(t, \cdot)\right\|_{2, x}^{2} \mathrm{~d} t\right] \leq C, \\
& \mathbb{E}\left[\int_{0}^{T}\left\|v_{N}(t, \cdot)\right\|_{2, x}^{2} \mathrm{~d} t\right] \leq C \Delta t,
\end{aligned}
$$

and

$$
\mathbb{E}\left[\int_{0}^{T}\left\|\nabla v_{N}(t, \cdot)\right\|_{2, x}^{2} \mathrm{~d} t\right] \leq C \Delta t
$$

Note in addition that, since $\left|m_{N}(t, x)\right|=1$ for a.e. $(\omega, t, x) \in \Omega \times[0, T] \times D$, one has

$$
\mathbb{E}\left[\int_{0}^{T}\left\|m_{N}(t, \cdot)\right\|_{2, x}^{2} \mathrm{~d} t\right] \leq C .
$$

\section{Tightness}

The aim of this section is to show that the sequence $\left(m_{N}\right)_{N \in \mathbb{N}^{*}}$ is tight. Applying then the classical Prokhorov and Skorohod theorems (see for instance [32]), we first get the relative compactness of the sequence of laws, and secondly we can assume the almost sure convergence to a certain limit $\bar{m}$, up to a change of probability space. In the sequel, we use the following notations : for all $t \in[0, T]$, we set

$$
X_{N}(t):=\sum_{0 \leq(n+1) \Delta t \leq t} m_{N}^{n} \times\left(G \Delta W_{N}^{n}\right)=\sum_{0 \leq(n+1) \Delta t \leq t} A_{N}^{n} \in L^{2}\left(\Omega ; H^{1}(D)^{3}\right)
$$


and

$$
X_{N}^{n}:=X_{N}(n \Delta t)=\sum_{0 \leq k \leq n-1} m_{N}^{k} \times\left(G \Delta W_{N}^{k}\right) .
$$

The process $t \mapsto X_{N}(t)$ is the martingale part of the semi-martingale $m_{N}$. It is a martingale with respect to a natural piecewise constant filtration, and corresponds to the noise induced fluctuations of the process $t \mapsto m_{N}(t)$. In order to get an almost sure convergence for the martingale part $X_{N}$, we consider the triplet $\left(m_{N}, X_{N}, G W\right)_{N \in \mathbb{N}^{*}}$, and show that it forms a tight sequence on a suitable space. This classical technique is used essentially to retrieve the noise term in this new probability space. This has the drawback that the new Wiener process depends on the integer $N \in \mathbb{N}^{*}$.

Proposition 2 The sequence

$$
\left(m_{N}, X_{N}, G W\right)_{N \in \mathbb{N} *}
$$

is tight in the space

$$
L^{2}\left([0, T] ; L^{2}(D)^{3}\right) \times L^{2}\left([0, T] ; L^{2}(D)^{3}\right) \times \mathcal{C}\left(0, T ; L^{2}(D)^{3}\right)
$$

The following result whose proof can be found e.g. in [33] will be needed for the proof of Proposition 2, which will be done later on.

Lemma 2 Let $B_{0} \subseteq B$ be two reflexive Banach spaces such that $B_{0}$ is compactly embedded in B. Let $\alpha>0$. Then the embedding

$$
L^{2}\left(0, T ; B_{0}\right) \cap H^{\alpha}(0, T ; B) \hookrightarrow L^{2}(0, T ; B)
$$

is compact.

We shall apply this lemma with $B=L^{2}(D)^{3}$, and $B_{0}=H^{1}(D)^{3}$. Therefore, in order to deduce the tightness, we need uniform $H^{\alpha}\left(0, T ; L^{2}(D)^{3}\right)$ estimates on $m_{N}$ and $X_{N}$ for some $\alpha>0$. These estimates are stated in the following proposition.

Proposition 3 For any $\alpha \in\left(0, \frac{1}{2}\right)$, there exists a constant $C=C\left(\|G\|_{2,2}, T, \alpha\right)$ such that

$$
\begin{aligned}
& \mathbb{E}\left[\left\|m_{N}\right\|_{H^{\alpha}\left(0, T ; L^{2}(D)^{3}\right)}^{2}\right] \leq C, \\
& \mathbb{E}\left[\left\|X_{N}\right\|_{H^{\alpha}\left(0, T ; L^{2}(D)^{3}\right)}^{2}\right] \leq C .
\end{aligned}
$$

Proof (Proposition 3.) We have to evaluate the following quantities for $\alpha \in\left(0, \frac{1}{2}\right)$ :

$$
\iint_{[0, T]^{2}} \frac{\mathbb{E}\left[\left\|m_{N}(t)-m_{N}(s)\right\|_{2, x}^{2}\right]}{|t-s|^{1+2 \alpha}} \mathrm{d} t \mathrm{~d} s,
$$


and

$$
\iint_{[0, T]^{2}} \frac{\mathbb{E}\left[\left\|X_{N}(t)-X_{N}(s)\right\|_{2, x}^{2}\right]}{|t-s|^{1+2 \alpha}} \mathrm{d} t \mathrm{~d} s .
$$

Notice that these integrals measure the regularity in time of the two processes $t \mapsto$ $m_{N}(t)$ and $t \mapsto X_{N}(t)$. Since $X_{N}$ is expected to be the martingale part in the canonical decomposition of the semi-martingale $m_{N}$, one expects $m_{N}$ to be at least as regular as $X_{N}$. In the sequel, we take $t, s \in[0, T]$, and assume without loss of generality that $t>s$. Thus, we first evaluate the following quantity:

$$
\mathbb{E}\left[\left\|X_{N}(t)-X_{N}(s)\right\|_{2, x}^{2}\right]=\mathbb{E}\left[\left\|\sum_{s<(n+1) \Delta t \leq t} A_{N}^{n}\right\|_{2, x}^{2}\right] .
$$

Observe that for $n \neq m$, the random variables $G \Delta W_{N}^{n}, G \Delta W_{N}^{m}$ are independent, with zero mean. Using also the fact that $m_{N}^{n}$ is independent of $G \Delta W_{N}^{k}$ for $1 \leq n \leq k \leq N$, Fubini's theorem, and the identity $a \cdot(b \times c)=a \times b \cdot c$, for $a, b, c \in \mathbb{R}^{3}$, one has for $m>n$,

$$
\begin{aligned}
\mathbb{E} & {\left[\left(A_{N}^{n}, A_{N}^{m}\right)_{2, x}\right] } \\
& =\int_{D} \mathbb{E}\left[\left(m_{N}^{n}(x) \times G \Delta W_{N}^{n}(x)\right) \cdot\left(m_{N}^{m}(x) \times G \Delta W_{N}^{m}(x)\right)\right] \mathrm{d} x \\
& =\int_{D} \mathbb{E}\left[\left(\left(m_{N}^{n}(x) \times G \Delta W_{N}^{n}(x)\right) \times m_{N}^{m}(x)\right)\right] \cdot \mathbb{E}\left[G \Delta W_{N}^{m}(x)\right] \mathrm{d} x \\
& =0 .
\end{aligned}
$$

Developing the sum (40) and using (15), one has

$$
\begin{aligned}
& \mathbb{E}\left[\left\|X_{N}(t)-X_{N}(s)\right\|_{2, x}^{2}\right] \\
& =\mathbb{E}\left[\sum_{s<(n+1) \Delta t \leq t}\left\|A_{N}^{n}\right\|_{2, x}^{2}\right]+2 \mathbb{E}\left[\sum_{\substack{s<(n+1) \Delta t \leq t \\
s<(m+1) \Delta t \leq t \\
n<m}}\left(A_{N}^{n}, A_{N}^{m}\right)_{2, x}\right] \\
& =\mathbb{E}\left[\sum_{s<(n+1) \Delta t \leq t}\left\|A_{N}^{n}\right\|_{2, x}^{2}\right] \leq C\|G\|_{2,0}^{2}\left(\sum_{s<(n+1) \Delta t \leq t} \Delta t\right) .
\end{aligned}
$$

We observe that the number of terms in the sum above is bounded by $\frac{t-s}{\Delta t}+1$, and deduce that for all $0 \leq s \leq t \leq T$, 


$$
\mathbb{E}\left[\left\|X_{N}(t)-X_{N}(s)\right\|_{2, x}^{2}\right] \leq C\|G\|_{2,0}^{2}(|t-s|+\Delta t) .
$$

Now, remark that (43) implies the uniform estimate of the $H^{\alpha}$ norm. Indeed, since $X_{N}$ is a piecewise constant function, the integrand $\mathbb{E}\left[\left\|X_{N}(t, \cdot)-X_{N}(s, \cdot)\right\|_{2, x}^{2}\right]$ vanishes for $(t, s) \in[n \Delta t,(n+1) \Delta t)^{2}$, for $0 \leq n \leq N-1$. Using moreover (43), there exists a constant $C=C\left(\|G\|_{2,0}, T\right)$ such that

$$
\begin{aligned}
& \iint_{[0, T]^{2}} \frac{\mathbb{E}\left[\left\|X_{N}(t)-X_{N}(s)\right\|_{2, x}^{2}\right]}{|t-s|^{1+2 \alpha}} \mathrm{d} t \mathrm{~d} s \\
& \quad \leq C \iint_{[0, T]^{2}} \frac{\mathrm{d} t \mathrm{~d} s}{|t-s|^{2 \alpha}}+C \Delta t \sum_{\substack{0 \leq m, n \leq N-1 \\
|n-m| \geq 1}} \int_{n \Delta t}^{(n+1) \Delta t} \int_{m \Delta t}^{(m+1) \Delta t} \frac{\mathrm{d} t \mathrm{~d} s}{|t-s|^{1+2 \alpha}} \\
& \quad=A+B .
\end{aligned}
$$

Since

$\bigcup_{\substack{0 \leq n, m \leq N-1 \\|n-m| \geq 2}}\left[n \Delta t,(n+1) \Delta t\left[\times\left[m \Delta t,(m+1) \Delta t\left[\subseteq\left\{(t, s) \in[0, T]^{2},|t-s|>\Delta t\right\}\right.\right.\right.\right.$,

we remark that

$$
B \leq \sum_{\substack{0 \leq m, n \leq N-1 \\|n-m|=1}} \int_{n \Delta t}^{(n+1) \Delta t} \int_{m \Delta t}^{(m+1) \Delta t} \frac{C \Delta t \mathrm{~d} t \mathrm{~d} s}{|t-s|^{1+2 \alpha}}+\iint_{\substack{[0, T]^{2} \\|t-s|>\Delta t}} \frac{C \Delta t}{|t-s|^{1+2 \alpha}} \mathrm{d} t \mathrm{~d} s .
$$

Finally, we get

$$
\begin{aligned}
& \iint_{[0, T]^{2}} \frac{\mathbb{E}\left[\left\|X_{N}(t)-X_{N}(s)\right\|_{2, x}^{2}\right]}{|t-s|^{1+2 \alpha}} \mathrm{d} t \mathrm{~d} s \\
& \leq 2 C \iint_{[0, T]^{2}} \frac{\mathrm{d} t \mathrm{~d} s}{|t-s|^{2 \alpha}}+2 C \Delta t \sum_{n=0}^{N-2} \int_{n \Delta t}^{(n+1) \Delta t} \int_{(n+1) \Delta t}^{(n+2) \Delta t} \frac{\mathrm{d} t \mathrm{~d} s}{|t-s|^{1+2 \alpha}} .
\end{aligned}
$$

The first term of the right hand side of (44) is bounded because $\alpha \in\left(0, \frac{1}{2}\right)$. Then, it is easy to show that

$$
\sum_{n=0}^{N-2} \int_{n \Delta t}^{(n+1) \Delta t} \int_{(n+1) \Delta t}^{(n+2) \Delta t} \frac{\mathrm{d} t \mathrm{~d} s}{|t-s|^{1+2 \alpha}}=O\left(\Delta t^{-2 \alpha}\right)
$$

and (39) is proved. 
We now turn to (38). Note that since we have already estimated $X_{N}$, it remains only to consider $m_{N}-X_{N}$. Using the definition of $m_{N}$ and $X_{N}$ together with (11), we write:

$$
\begin{aligned}
& \left(m_{N}(t)-X_{N}(t)\right)-\left(m_{N}(s)-X_{N}(s)\right) \\
& =\sum_{s<(n+1) \Delta t \leq t}\left(m_{N}^{n+1}-m_{N}^{n}-A_{N}^{n}\right) \\
& =\sum_{s<(n+1) \Delta t \leq t}\left(\frac{m_{N}^{n}+A_{N}^{n}}{\left|m_{N}^{n}+A_{N}^{n}\right|}-m_{N}^{n}-A_{N}^{n}\right) \\
& \quad+\sum_{s<(n+1) \Delta t \leq t}\left(\frac{m_{N}^{n}+v_{N}^{n}}{\left|m_{N}^{n}+v_{N}^{n}\right|}-\frac{m_{N}^{n}+A_{N}^{n}}{\left|m_{N}^{n}+A_{N}^{n}\right|}\right) .
\end{aligned}
$$

Then, taking the $L^{2}(D)^{3}$ norm, and the expectation, we get

$$
\begin{aligned}
& \mathbb{E}\left[\left\|m_{N}(t)-X_{N}(t)-\left(m_{N}(s)-X_{N}(s)\right)\right\|_{2, x}^{2}\right] \\
& \leq 2 \mathbb{E}\left[\left\|\sum_{s<(n+1) \Delta t \leq t} \frac{m_{N}^{n}+A_{N}^{n}}{\left|m_{N}^{n}+A_{N}^{n}\right|}-m_{N}^{n}-A_{N}^{n}\right\|_{2, x}^{2}\right] \\
& \quad+2 \mathbb{E}\left[\left\|\sum_{s<(n+1) \Delta t \leq t} \frac{m_{N}^{n}+v_{N}^{n}}{\left|m_{N}^{n}+v_{N}^{n}\right|}-\frac{m_{N}^{n}+A_{N}^{n}}{\left|m_{N}^{n}+A_{N}^{n}\right|}\right\|_{2, x}^{2}\right] .
\end{aligned}
$$

For the first term in the right hand side of (47), observe that for any $m, V \in \mathbb{R}^{3}$, s.t. $V \perp m$ and $|m|=1$, one has :

$$
\left|\frac{m+V}{|m+V|}-m-V\right| \leq \sqrt{1+|V|^{2}}-1 \leq \frac{1}{2}|V|^{2} .
$$

Using Cauchy-Schwarz inequality, and (48) on each term of the sum (remember that $A_{N}^{n} \perp m_{N}^{n}$, see (14)), one has:

$$
\begin{aligned}
& E\left[\sum_{s<(n+1) \Delta t \leq t} \frac{m_{N}^{n}+A_{N}^{n}}{\left|m_{N}^{n}+A_{N}^{n}\right|}-m_{N}^{n}-A_{N}^{n} \|_{2, x}^{2}\right] \\
& \leq\left(\frac{t-s}{\Delta t}+1\right) \sum_{s<(n+1) \Delta t \leq t} \mathbb{E}\left[\left\|\frac{m_{N}^{n}+A_{N}^{n}}{\left|m_{N}^{n}+A_{N}^{n}\right|}-m_{N}^{n}-A_{N}^{n}\right\|_{2, x}^{2}\right] \\
& \leq \frac{1}{4}\left(\frac{t-s}{\Delta t}+1 \sum_{s<(n+1) \Delta t \leq t} \mathbb{E}\left[\left\|A_{N}^{n}\right\|_{4, x}^{4}\right] .\right.
\end{aligned}
$$


Then we have by (16):

$$
E\left[\left\|\sum_{s<(n+1) \Delta t \leq t} \frac{m_{N}^{n}+A_{N}^{n}}{\left|m_{N}^{n}+A_{N}^{n}\right|}-m_{N}^{n}-A_{N}^{n}\right\|_{2, x}^{2}\right] \leq C\left(\|G\|_{2,1}, T\right)(|t-s|+\Delta t)^{2}
$$

Similarly, for the second term in (49), we use the fact that the map $x \mapsto \frac{x}{|x|}$, is 1-Lipschitz for $|x| \geq 1$, together with Cauchy-Schwarz inequality and (18). Then

$$
\begin{aligned}
& \mathbb{E}\left[\left\|\sum_{s<(n+1) \Delta t \leq t} \frac{m_{N}^{n}+v_{N}^{n}}{\left|m_{N}^{n}+v_{N}^{n}\right|}-\frac{m_{N}^{n}+A_{N}^{n}}{\left|m_{N}^{n}+A_{N}^{n}\right|}\right\|_{2, x}^{2}\right] \\
& \leq\left(\frac{t-s}{\Delta t}+1\right) \mathbb{E}\left[\sum_{s<(n+1) \Delta t \leq t}\left\|v_{N}^{n}-A_{N}^{n}\right\|_{2, x}^{2}\right] \\
& \leq C\left(\|G\|_{2,2}, T\right)(|t-s|+\Delta t) .
\end{aligned}
$$

Using (49) and (50) in (47), together with (39), we conclude that there exists a constant $C=C\left(\|G\|_{2,2}, T\right)$, independent of $N \in \mathbb{N}^{*}$, such that

$$
\mathbb{E}\left[\left\|m_{N}(t, \cdot)-m_{N}(s, \cdot)\right\|_{2, x}^{2}\right] \leq C(|t-s|+\Delta t) .
$$

We have proved the same inequality as for the process $X_{N}$ (see (43)), thus the conclusion follows in the same way as before.

We now turn to the proof of Proposition 2.

Proof (Proposition 2) Remark that thanks to (35) and (31), $\left(m_{N}\right)_{N \in \mathbb{N}^{*}}$ is bounded in $L^{2}\left(\Omega \times[0, T] ; H^{1}(D)^{3}\right)$. Let us prove the same for the process $X_{N}$. One has:

$$
\begin{aligned}
\mathbb{E}\left[\left\|X_{N}\right\|_{L^{2}\left(0, T ; L^{2}(D)^{3}\right)}^{2}\right]= & \mathbb{E}\left[\sum_{n=0}^{N-1}\left\|X_{N}^{n}\right\|_{2, x}^{2} \Delta t\right] \\
= & \sum_{n=0}^{N-1} \Delta t \mathbb{E}\left[\left\|\sum_{k \leq n} A_{N}^{k}\right\|_{2, x}^{2}\right] \\
= & \sum_{n=0}^{N-1} \Delta t\left(\mathbb{E}\left[\sum_{k \leq n}\left\|A_{N}^{k}\right\|_{2, x}^{2}\right]\right. \\
& \left.+2 \mathbb{E}\left[\sum_{0 \leq k<l \leq n}\left(A_{N}^{k}, A_{N}^{l}\right)_{2, x}\right]\right) .
\end{aligned}
$$

As before, the second term vanishes [see (42)], while the first term is bounded by $C\left(\|G\|_{2,0}, T\right)$ thanks to (15). 
Similarly, for $k \neq l$, we have $\mathbb{E}\left[\left(\nabla A_{N}^{k}, \nabla A_{N}^{l}\right)_{2, x}\right]=0$. Moreover, using (27), we get

$$
\begin{aligned}
\mathbb{E}\left[\left\|\nabla X_{N}\right\|_{2, x}^{2}\right] & =\sum_{n=0}^{N-1} \Delta t \sum_{k \leq n} \mathbb{E}\left[\left\|\nabla A_{N}^{k}\right\|_{2, x}^{2}\right] \\
& \leq C\|G\|_{2,2}^{2} \sum_{n=0}^{N-1} \Delta t \sum_{k \leq n} \Delta t \\
& \leq C^{\prime}\left(\|G\|_{2,2}, T\right) .
\end{aligned}
$$

Therefore, there exists a constant $C=C\left(\|G\|_{2,2}, T\right)>0$ such that

$$
\mathbb{E}\left[\left\|X_{N}\right\|_{L^{2}\left(0, T ; H^{1}(D)^{3}\right)}^{2}\right] \leq C \text {. }
$$

The tightness of the sequence $\left(m_{N}, X_{N}, W\right)$ is now obtained in a classical way. Let $R>0$, and fix $\alpha \in\left(0, \frac{1}{2}\right)$. We consider the product space

$$
\left.E:=L^{2}\left(0, T ; L^{2}(D)^{3}\right) \times L^{2}\left(0, T ; L^{2}(D)^{3}\right)\right) \times \mathcal{C}\left([0, T] ; L^{2}(D)^{3}\right)
$$

endowed with its classical product norm. Thanks to Lemma 2, and a standard Ascoli compactness theorem, the space

$$
\begin{aligned}
F:= & L^{2}\left(0, T ; H^{1}(D)^{3}\right) \times L^{2}\left(0, T ; H^{1}(D)^{3}\right) \times \mathcal{C}\left([0, T] ; H^{1}(D)^{3}\right) \\
& \bigcap H^{\alpha}\left(0, T ; L^{2}(D)^{3}\right) \times H^{\alpha}\left(0, T ; L^{2}(D)^{3}\right) \times \mathcal{C}^{\alpha}\left([0, T] ; L^{2}(D)^{3}\right)
\end{aligned}
$$

is compactly embedded in $E$. Using Markov inequality, one has

$$
\begin{aligned}
& \mathbb{P}\left(\left(m_{N}, X_{N}, G W\right) \notin B_{F}(0, R)\right) \\
& \quad \leq \frac{1}{R^{2}}\left(\mathbb{E}\left[\left\|m_{N}\right\|_{L^{2}\left(0, T ; H^{1}\right)}^{2}\right]+\mathbb{E}\left[\left\|m_{N}\right\|_{H^{\alpha}\left([0, T] ; L^{2}\right)}^{2}\right]+\mathbb{E}\left[\left\|X_{N}\right\|_{L^{2}\left(0, T ; H^{1}\right)}^{2}\right]\right. \\
& \left.\quad+\mathbb{E}\left[\left\|X_{N}\right\|_{H^{\alpha}\left([0, T] ; L^{2}\right)}^{2}\right]+\mathbb{E}\left[\|G W\|_{\mathcal{C}^{\alpha}\left([0, T] ; H^{1}\right)}^{2}\right]\right)
\end{aligned}
$$

Then, using the bounds (38), and (39), (8), the classical properties of a $G G^{*}$-Wiener process and also (17), the right hand side of (51) tends to 0 as $R \rightarrow \infty$ uniformly in $N \in$ $\mathbb{N}^{*}$. Since the sets $B_{F}(0, R)$ are precompacts in $E$, the sequence $\left(m_{N}, X_{N}, G W\right)_{N \in \mathbb{N}^{*}}$ is tight in $E$, and the proposition is proved.

A simple application of Prokhorov and Skorohod theorem leads to the following corollary:

Corollary 1 There exists a new probability space $(\bar{\Omega}, \overline{\mathcal{F}}, \overline{\mathbb{P}})$, a sequence of random variables on this space $\left(\bar{m}_{N}, \bar{X}_{N}, G \bar{W}_{N}\right)_{N \in \mathbb{N}^{*}}$ taking its values in the space $L^{2}\left(0, T ; L^{2}(D)^{3}\right) \times L^{2}\left(0, T ; L^{2}(D)^{3}\right) \times \mathcal{C}\left(0, T ; L^{2}(D)^{3}\right)$, with the same laws, 
for each $N \in \mathbb{N}^{*}$, as $\left(m_{N}, X_{N}, G W\right)$, and a triplet $(\bar{m}, \bar{X}, G \bar{W})$ of r.v. in $L^{2}\left(0, T ; L^{2}(D)^{3}\right) \times L^{2}\left(0, T ; L^{2}(D)^{3}\right) \times \mathcal{C}\left(0, T ; L^{2}(D)^{3}\right)$, so that up to a subsequence,

$$
\begin{aligned}
& \bar{m}_{N} \underset{N \rightarrow \infty}{\longrightarrow} \bar{m} \text { a.s. in } L^{2}\left([0, T] ; L^{2}(D)^{3}\right), \\
& \bar{X}_{N} \underset{N \rightarrow \infty}{\longrightarrow} \bar{X} \text { a.s. in } L^{2}\left([0, T] ; L^{2}(D)^{3}\right), \\
& G \bar{W}_{N} \underset{N \rightarrow \infty}{\longrightarrow} G \bar{W} \quad \text { a.s.in } \mathcal{C}\left(0, T ; L^{2}(D)^{3}\right) .
\end{aligned}
$$

Since $m_{N}, X_{N}$ are piecewise constant processes, the same is also true for their counterparts in the new probability space $\bar{\Omega}$. We define the following discrete parameter processes, for $0 \leq n \leq N$ :

$$
\begin{aligned}
\bar{m}_{N}^{n} & :=\bar{m}_{N}(n \Delta t) \in L^{2}(D)^{3}, \\
\bar{X}_{N}^{n} & :=\bar{X}_{N}(n \Delta t) \in L^{2}(D)^{3},
\end{aligned}
$$

and also

$$
\begin{aligned}
G \Delta \bar{W}_{N}^{n} & :=G \bar{W}_{N}((n+1) \Delta t)-G \bar{W}_{N}(n \Delta t), \\
\bar{A}_{N}^{n} & :=\bar{m}_{N}^{n} \times\left(G \Delta \bar{W}_{N}^{n}\right),
\end{aligned}
$$

and $\bar{v}_{N}^{n}$ as the unique solution of (10) associated to the data $\left(\bar{m}_{N}^{n}, G \Delta \bar{W}_{N}^{n}\right)$, i.e. for all $0 \leq n \leq N-1$, and all $\varphi \in \overline{\mathbb{W}}_{N, n}$,

$$
\begin{aligned}
& \left(\bar{v}_{N}^{n}-\bar{m}_{N}^{n} \times \bar{v}_{N}^{n}, \varphi\right)_{2, x}+2 \theta \Delta t\left(\nabla \bar{v}_{N}^{n}, \nabla \varphi\right)_{2, x} \\
& =-2 \Delta t\left(\nabla \bar{m}_{N}^{n}, \nabla \varphi\right)_{2, x}+\left(\left(\operatorname{Id}-\bar{m}_{N}^{n} \times\right)\left(\bar{m}_{N}^{n} \times G \Delta \bar{W}_{N}^{n}\right), \varphi\right)_{2, x} \\
& \quad+\frac{\Delta t}{2} \sum_{i \in \mathbb{N}}\left(\left(\operatorname{Id}-\bar{m}_{N}^{n} \times\right)\left(\left(\bar{m}_{N}^{n} \times G_{i}\right) \times G_{i}\right), \varphi\right)_{2, x},
\end{aligned}
$$

where

$$
\overline{\mathbb{W}}_{N, n}(\omega):=\left\{\psi \in H^{1}(D)^{3}, \forall x \in D, \psi(x) \perp \bar{m}_{N}^{n}(\omega, x)\right\}
$$

These random variables have the same laws as their counterparts in $\Omega$ that is (respectively) $m_{N}^{n}, X_{N}^{n}, G \Delta W_{N}^{n}$ and $A_{N}^{n}:=m_{N}^{n} \times\left(G \Delta W_{N}^{n}\right)$. We already noticed that $v_{N}^{n}$ depends continuously on the couple $\left(m_{N}^{n}, G \Delta \bar{W}_{N}^{n}\right)$ through (10), and thus the law of $\bar{v}_{N}^{n}$ is the same as the law of $v_{N}^{n}$. It also follows that we have the identity

$$
\bar{m}_{N}^{n+1}=\frac{\bar{m}_{N}^{n}+\bar{v}_{N}^{n}}{\left|\bar{m}_{N}^{n}+\bar{v}_{N}^{n}\right|} \quad \text { a.s. }
$$


We still need to define the following processes on $\bar{\Omega}: \forall t \in[0, T]$,

$$
\bar{v}_{N}(t):=\bar{v}_{N}^{n} \text { if } t \in[n \Delta t,(n+1) \Delta t),
$$

and

$$
\bar{w}_{N}(t):=\frac{\bar{v}_{N}^{n}-\bar{A}_{N}^{n}}{\Delta t} \text { if } t \in[n \Delta t,(n+1) \Delta t) .
$$

Remark 4 By (31) and a classical compactness argument, we may assume that up a subsequence the following convergence holds

$$
\nabla \bar{m}_{N} \underset{N \rightarrow \infty}{\rightarrow} \nabla \bar{m} \quad \text { weakly in } L^{2}(\bar{\Omega} \times[0, T] \times D)^{3 \times 3} .
$$

\section{Convergence of the martingale part}

In Sect. 4 we proved that the process $\bar{X}_{N}$ converges almost surely in $L^{2}([0, T] \times D)^{3}$ to $\bar{X}$. Here we show that $\bar{X}$ defines a square integrable continuous martingale with values in $L^{2}(D)^{3}$. We define the filtration $\left(\overline{\mathcal{F}}_{t}\right)_{t \in[0, T]}$ as

$$
\overline{\mathcal{F}}_{t}=\sigma\{G \bar{W}(s), s \leq t\}
$$

Proposition 4 The process $t \in[0, T] \mapsto \bar{X}(t, \omega) \in L^{2}(D)^{3}$ is a square integrable continuous martingale with respect to the filtration $\left(\overline{\mathcal{F}}_{t}\right)$, with quadratic variation defined for all $a, b \in L^{2}(D)^{3}$ by:

$$
\left(\ll \bar{X} \gg_{t} a, b\right)_{2, x}=\int_{0}^{t}(\bar{m} \times(G a), \bar{m} \times(G b))_{2, x} \mathrm{~d} s .
$$

The proof needs an additional martingale-type uniform estimate on $X_{N}$.

Proposition 5 For all $q \in \mathbb{N}$, there exists a constant $C=C\left(\|G\|_{2,0}, T, q\right)>0$ independent of $N \in \mathbb{N}^{*}$, such that

$$
\mathbb{E}\left[\max _{n \in\{0, \ldots, N\}}\left\|\bar{X}_{N}^{n}\right\|_{2, x}^{2 q}\right] \leq C .
$$

To prove proposition 5, we state a discrete version of the Burkholder-Davis-Gundy inequality with values in a Hilbert space. The following result is a particular case of Proposition 2 of [34], and we therefore omit the proof.

Lemma 3 For a given discrete parameter martingale $\left(M^{n}\right)_{0 \leq n \leq N}$ with values in a Hilbert space $H$, for any $q \in \mathbb{N}^{*}$, there exist $C=C(q)>0$ such that the following inequality holds :

$$
\mathbb{E}\left[\max _{0 \leq n \leq N}\left\|M^{n}\right\|_{H}^{2 q}\right] \leq C \mathbb{E}\left[\left(\sum_{n=0}^{N-1}\left\|M^{n+1}-M^{n}\right\|_{H}^{2}\right)^{q}\right] .
$$


Remark 5 Since for all $N \in \mathbb{N}^{*}$, the laws of $X_{N}$ and $\bar{X}_{N}$ are equal, note that for all $t \in[0, T]$, and almost surely,

$$
\bar{X}_{N}(t)=\sum_{0 \leq(n+1) \Delta t \leq t} \bar{m}_{N}^{n} \times G \Delta \bar{W}_{N}^{n}
$$

It is easily seen, using (52) and (53) that $\left(\bar{m}_{N}^{n}\right)_{0 \leq n \leq N}$ is adapted to

$$
\overline{\mathbb{F}}_{N}^{n}=\sigma\left\{G \bar{W}_{N}(k \Delta t) ; k \in \mathbb{N}^{*}, k \leq n\right\},
$$

and the process $\bar{X}_{N}^{n}$ defines a martingale with respect to this filtration. In particular, we have the following identity : for all $0 \leq n \leq n^{\prime} \leq N$, and any bounded continuous function $\phi$ on $\left(L^{2}(D)^{3}\right)^{n}$,

$$
\mathbb{E}\left[\left(\bar{X}_{N}^{n^{\prime}}-\bar{X}_{N}^{n}\right) \phi\left(G \bar{W}_{N}(\Delta t), \ldots, G \bar{W}_{N}(n \Delta t)\right)\right]=0
$$

The reader may also check that for any $n, n^{\prime}, \phi$ as above, and for all $a, b \in L^{2}(D)^{3}$,

$$
\begin{aligned}
\mathbb{E}[( & \left(\bar{X}_{N}^{n^{\prime}}, a\right)_{2, x}\left(\bar{X}_{N}^{n^{\prime}}, b\right)_{2, x}-\left(\bar{X}_{N}^{n}, a\right)_{2, x}\left(\bar{X}_{N}^{n}, b\right)_{2, x} \\
- & \left.\sum_{n \leq k \leq n^{\prime}-1} \Delta t\left(\bar{m}_{N}^{k} \times(G a), \bar{m}_{N}^{k} \times(G b)\right)_{2, x}\right) \\
& \left.\phi\left(G \bar{W}_{N}(\Delta t), \ldots, G \bar{W}_{N}(n \Delta t)\right)\right]=0 .
\end{aligned}
$$

Equation (61) gives us the quadratic variation of $\left(\bar{X}_{N}^{n}\right)_{0 \leq n \leq N}$.

Proof (Proposition 5) Assume that $N \in \mathbb{N}^{*}$ is given. We apply Lemma 3 to the discrete parameter martingale $\left(\bar{X}_{N}^{n}\right)_{0<n<N}$, which takes values in the Hilbert space $H=L^{2}(D)^{3}$. Thanks to (58), and Hölder's inequality, one has

$$
\begin{aligned}
\mathbb{E}\left[\left(\sum_{n=0}^{N-1}\left\|\bar{X}_{N}^{n+1}-\bar{X}_{N}^{n}\right\|_{2, x}^{2}\right)^{q}\right] & =\mathbb{E}\left[\left(\sum_{n=0}^{N-1}\left\|\bar{m}_{N}^{n} \times\left(G \Delta \bar{W}_{N}^{n}\right)\right\|_{2, x}^{2}\right)^{q}\right] \\
& \leq N^{q-1} \sum_{n=0}^{N-1} \mathbb{E}\left[\left\|\bar{m}_{N}^{n} \times\left(G \Delta \bar{W}_{N}^{n}\right)\right\|_{2, x}^{2 q}\right]
\end{aligned}
$$

It is known (see for instance [32], corollary 2.17 ) that since $G \Delta \bar{W}_{N}^{n}$ is a gaussian random variable with covariance $\Delta t G G^{*}$, there exists a constant $C\left(\|G\|_{2,0}, q\right)>0$ (independent of $n$ and $N$ ) such that :

$$
\mathbb{E}\left[\left\|G \Delta \bar{W}_{N}^{n}\right\|_{2, x}^{2 q}\right] \leq C\left(\|G\|_{2,0}, q\right) \Delta t^{q}
$$


Thus one has, recalling that $\left|\bar{m}_{N}^{n}\right|=1$ a.e.,

$$
\mathbb{E}\left[\left(\sum_{n=0}^{N-1}\left\|\bar{X}_{N}^{n+1}-\bar{X}_{N}^{n}\right\|_{2, x}^{2}\right)^{q}\right] \leq N\left(C\left(\|G\|_{2,0}, q\right) \Delta t^{q}\right) N^{q-1} \leq C^{\prime}\left(\|G\|_{2,0}, T, q\right) .
$$

This proves proposition 5 .

We now turn to the proof of Proposition 4.

Proof (Proposition 4.) $\bar{X}$ is a martingale:

We use equalities (60) and (61). We have to show that for any bounded continuous function $\phi$ defined on the space $\left(L^{2}(D)^{3}\right)^{K}$, any $a, b \in L^{2}(D)^{3}$ the following relations hold for almost all $0 \leq s \leq t \leq T$, all $K \in \mathbb{N}^{*}$, and $t_{1} \leq \ldots t_{K}<s$ :

$$
\mathbb{E}\left[(\bar{X}(t)-\bar{X}(s)) \phi\left(G \bar{W}\left(t_{1}\right), \ldots, G \bar{W}\left(t_{K}\right)\right)\right]=0
$$

and

$$
\begin{aligned}
& \mathbb{E}\left[\left((\bar{X}(t), a)_{2, x}(\bar{X}(t), b)_{2, x}-(\bar{X}(s), a)_{2, x}(\bar{X}(s), b)_{2, x}\right.\right. \\
& \left.\left.-\int_{s}^{t}(\bar{m}(\sigma) \times(G a), \bar{m}(\sigma) \times(G b))_{2, x} \mathrm{~d} \sigma\right) \phi\left(G \bar{W}\left(t_{1}\right), \ldots, G \bar{W}\left(t_{K}\right)\right)\right]=0 .
\end{aligned}
$$

First, observe that as a consequence of Proposition 5, and Egorov's Theorem,

$$
\bar{X}_{N} \underset{N \rightarrow \infty}{\longrightarrow} \bar{X} \text { in } L^{2}(\bar{\Omega} \times[0, T] \times D)^{3} .
$$

Hence, up to a subsequence, one has for almost all $t, s \in[0, T]$,

$$
\bar{X}_{N}(t)-\bar{X}_{N}(s) \underset{N \rightarrow \infty}{\longrightarrow} \bar{X}(t)-\bar{X}(s), \quad \text { in } L^{2}(\bar{\Omega} \times D)^{3} .
$$

For all $0 \leq k \leq K$, if $\left[\frac{t_{k}}{\Delta t}\right]$ denotes the floor of $\frac{t_{k}}{\Delta t}$, then $\left[\frac{t_{k}}{\Delta t}\right] \Delta t$ tends to $t_{k}$ as $N \rightarrow \infty$. Taking into account the almost sure continuity of the limit process $G \bar{W}$, and the fact that the process $G \bar{W}_{N}$ converges almost surely to $G \bar{W}$ in $\mathcal{C}\left([0, T] ; L^{2}(D)^{3}\right)$ as $N$ tends to $\infty$, one has

$$
\begin{aligned}
& \left(G \bar{W}_{N}\left(\left[\frac{t_{1}}{\Delta t}\right] \Delta t\right), \ldots, G \bar{W}_{N}\left(\left[\frac{t_{K}}{\Delta t}\right] \Delta t\right)\right) \\
& \quad \underset{N \rightarrow \infty}{\longrightarrow}\left(G \bar{W}\left(t_{1}\right), \ldots, G \bar{W}\left(t_{K}\right)\right) \text { in }\left(L^{2}(D)^{3}\right)^{K} .
\end{aligned}
$$

The application $\phi$ being continuous, we conclude that

$$
\begin{gathered}
\mathbb{E}\left[\left(\bar{X}_{N}(t)-\bar{X}_{N}(s)\right) \phi\left(G \bar{W}_{N}\left(\left[\frac{t_{1}}{\Delta t}\right] \Delta t\right), \ldots, G \bar{W}_{N}\left(\left[\frac{t_{K}}{\Delta t}\right] \Delta t\right)\right)\right] \\
\underset{N \rightarrow \infty}{\longrightarrow} \mathbb{E}\left[\left(\bar{X}_{N}(t)-\bar{X}_{N}(s)\right) \phi\left(G \bar{W}\left(t_{1}\right), \ldots, G \bar{W}\left(t_{K}\right)\right)\right] .
\end{gathered}
$$


On the other hand, by (60)

$$
\mathbb{E}\left[\left(\bar{X}_{N}(t)-\bar{X}_{N}(s)\right) \phi\left(G \bar{W}_{N}\left(\left[\frac{t_{1}}{\Delta t}\right] \Delta t\right), \ldots, G \bar{W}_{N}\left(\left[\frac{t_{K}}{\Delta t}\right] \Delta t\right)\right)\right]=0,
$$

and (63) is proved. If $a, b \in L^{2}([0, T] \times D)^{3}$, then (61) implies that:

$$
\begin{gathered}
\mathbb{E}\left[\left(\left(\bar{X}_{N}(t), a\right)_{2, x}\left(\bar{X}_{N}(t), b\right)_{2, x}-\left(\bar{X}_{N}(s), a\right)_{2, x}\left(\bar{X}_{N}(s), b\right)_{2, x}\right.\right. \\
\left.-\sum_{s<(n+1) \Delta t \leq t} \Delta t\left(\bar{m}_{N}^{n} \times(G a), \bar{m}_{N}^{n} \times(G b)\right)_{2, x}\right) \\
\left.\phi\left(G \bar{W}_{N}\left(\left[\frac{t_{1}}{\Delta t}\right] \Delta t\right), \ldots, G \bar{W}_{N}\left(\left[\frac{t_{K}}{\Delta t}\right] \Delta t\right)\right)\right]=0 .
\end{gathered}
$$

Moreover,

$$
\left(\bar{X}_{N}(t), a\right)_{2, x}\left(\bar{X}_{N}(t), b\right)_{2, x}-\left(\bar{X}_{N}(s), a\right)_{2, x}\left(\bar{X}_{N}(s), b\right)_{2, x}
$$

tends to

$$
(\bar{X}(t), a)_{2, x}(\bar{X}(t), b)_{2, x}-(\bar{X}(s), a)_{2, x}(\bar{X}(s), b)_{2, x}
$$

in $L^{1}(\bar{\Omega})$, while

$$
\sum_{s<(n+1) \Delta t \leq t} \Delta t\left(\bar{m}_{N}^{n} \times(G a), \bar{m}_{N}^{n} \times(G b)\right)_{2, x}
$$

converges to

$$
\int_{s}^{t}(\bar{m}(\sigma) \times G a, \bar{m}(\sigma) \times G b)_{2, x} \mathrm{~d} \sigma,
$$

in $L^{1}(\bar{\Omega})$. This proves (64). It remains to prove that $\bar{X}$ has continuous trajectories.

Proof of the continuity.

We prove that the limit $\bar{X}$ satisfies the assumptions of Kolmogorov's test (see e.g. [32], Theorem 3.3). More precisely, we show that for any $q \in \mathbb{N}^{*}$, there exists $C_{q}>0$, such that for almost every $(t, s) \in[0, T]^{2}$,

$$
\mathbb{E}\left[\|\bar{X}(t)-\bar{X}(s)\|_{2, x}^{2 q}\right] \leq C_{q}|t-s|^{q} .
$$

Let $T \geq t>s \geq 0, \quad$ and $n, n^{\prime} \in \mathbb{N}$, the unique integers such that $t \in\left[n^{\prime} \Delta t,\left(n^{\prime}+\right.\right.$ 1) $\Delta t)$ and $s \in\left[n \Delta t,(n+1) \Delta t\left[\right.\right.$. One has $\left|t-n^{\prime} \Delta t\right| \leq \Delta t$ and $|s-n \Delta t| \leq \Delta t$. We consider the discrete parameter martingale which starts at $n \Delta t$, and whose increments are the same as $\left(\bar{X}_{N}^{k}\right)_{k \in\{0, \ldots, N\}}$. More precisely, let $\left(M_{N}^{l}\right)_{0 \leq l \leq n^{\prime}-n}$ be the discrete parameter process defined by

$$
\bar{M}_{N}^{l}=\bar{X}_{N}^{n+l}-\bar{X}_{N}^{n}=\sum_{k=n+1}^{n+l} \bar{A}_{N}^{k}, \quad \text { for } \quad 0 \leq l \leq n^{\prime}-n .
$$


The process $\left(\bar{M}_{N}^{l}\right)_{0 \leq l \leq n^{\prime}-n}$ defines a martingale for the discrete filtration $\left(\overline{\mathbb{F}}_{(l+n) \Delta t}\right)_{0 \leq l \leq n^{\prime}-n}$, [see (12)]. Using similar arguments as for the proof of Proposition 5, and in particular Lemma 3,

$$
\begin{aligned}
\mathbb{E}\left[\left\|\bar{X}_{N}(t)-\bar{X}_{N}(s)\right\|_{2, x}^{2 q}\right] & \leq \mathbb{E}\left[\max _{l=0, \ldots, n^{\prime}-n}\left\|\bar{M}_{N}^{l}\right\|_{2, x}^{2 q}\right] \\
& \leq C \sum_{k=n+1}^{n^{\prime}} \mathbb{E}\left[\left\|\bar{A}_{N}^{k}\right\|_{2, x}^{2 q}\right]\left(n^{\prime}-n\right)^{q-1} \\
& \leq C\left(\|G\|_{2,0}, q\right)\left(n^{\prime} \Delta t-n \Delta t\right)^{q} \\
& \leq C\left(\|G\|_{2,0}, q\right)(|t-s|+\Delta t)^{q} .
\end{aligned}
$$

Then, (66) follows from (65) and Fatou's Lemma. Thus, $\bar{X}$ defines a continuous martingale with respect to $\left(\overline{\mathcal{F}}_{t}\right)_{t \in[0, T]}$ [see (57)]. As we saw in the proof of Proposition 2 the processes $\bar{X}_{N}$, for $N \in \mathbb{N}^{*}$ are square-integrable, uniformly in $N$, thus the almost sure limit $\bar{X}$ is square-integrable. This proves Proposition 4.

We are now ready to apply the continuous martingale representation theorem for Hilbert space-valued Wiener processes. We have shown that the limit process $\bar{X}$ satisfies its hypotheses. The quadratic variation of $\bar{X}$ is given, for any $a, b \in L^{2}(D)^{3}$, by :

$$
\left(\ll \bar{X} \gg_{t} a, b\right)_{2, x}=\int_{0}^{t}(\bar{m}(s) \times(G a), \bar{m}(s) \times(G b))_{2, x} \mathrm{~d} s, \quad t \in[0, T] .
$$

There exists an enlarged probability space $(\tilde{\Omega}, \tilde{\mathcal{F}}, \tilde{\mathbb{P}})$, with $\bar{\Omega} \subseteq \tilde{\Omega}$, a filtration $\left\{\tilde{\mathcal{F}}_{t}\right\}$, and a $L^{2}(D)^{3}$-valued Wiener process $G \tilde{W}$ defined on $(\tilde{\Omega}, \tilde{\mathcal{F}}, \tilde{\mathbb{P}})$, such that $\bar{X}, \bar{m}$ can be extended to random variables on this space, and

$$
\bar{X}(t, \tilde{\omega})=\int_{0}^{t} \bar{m}(s, \tilde{\omega}) \times G d \tilde{W}(s, \tilde{\omega})
$$

\section{Identification of the limit}

In this section, the purpose is to find a relation between $\bar{X}$ and the limit $\bar{m}$. Noticing that $\sum_{0 \leq(n+1) \Delta t \leq t}\left(\bar{m}_{N}^{n+1}-\bar{m}_{N}^{n}\right)=\bar{m}_{N}(t)-m_{0}$, and by definition of $\bar{w}_{N}^{n}$ in (55), one may write $\bar{X}_{N}(t)$ as :

$$
\bar{X}_{N}(t)=\bar{m}_{N}(t)-m_{0}-\sum_{0 \leq(n+1) \Delta t \leq t} \Delta t \bar{w}_{N}^{n}-\sum_{0 \leq(n+1) \Delta t \leq t}\left(\bar{m}_{N}^{n+1}-\bar{m}_{N}^{n}-\bar{v}_{N}^{n}\right)
$$

for any $t \in[0, T]$. 
Proposition 6 Up to a subsequence:

$$
\bar{w}_{N} \underset{N \rightarrow \infty}{\rightarrow} \bar{w} \text { weakly in } L^{2}(\bar{\Omega} \times[0, T] \times D)^{3},
$$

with

$$
\bar{w}=\Delta \bar{m}+\bar{m}|\nabla \bar{m}|^{2}+\bar{m} \times \Delta \bar{m}+\frac{1}{2} \Pi_{\bar{m} \perp} \sum_{i \in \mathbb{N}}\left(\bar{m} \times G_{i}\right) \times G_{i},
$$

and $\Pi_{\bar{m}(\omega, t, x)^{\perp}}$ stands for the $\mathbb{R}^{3}$ orthogonal projection on $\bar{m}(\omega, t, x)^{\perp}$, for each $\omega, t, x$.

Corollary 2 Up to a subsequence, for anyt $\in[0, T], \sum_{0 \leq(n+1) \Delta t \leq t} \Delta t \bar{w}_{N}^{n}$ converges weakly in $L^{2}(\bar{\Omega} \times[0, T] \times D)^{3}$ to

$$
\begin{aligned}
& \int_{0}^{t}\left(\Delta \bar{m}(s)+\bar{m}(s)|\nabla \bar{m}(s)|^{2}+\bar{m}(s) \times \Delta \bar{m}(s)\right. \\
& \left.+\frac{1}{2} \Pi_{\bar{m}(s)^{\perp}} \sum_{i \in \mathbb{N}}\left(\bar{m}(s) \times G_{i}\right) \times G_{i}\right) \mathrm{d} s .
\end{aligned}
$$

Proof (Proposition 6.) Thanks to (32), the equality of the laws of $w_{N}$ and $\bar{w}_{N}$, and Alaoglu theorem, we can assume that up to a subsequence, $\bar{w}_{N}$ converges weakly to a limit $\bar{w}$ in $L^{2}(\bar{\Omega} \times[0, T] \times D)^{3}$. Because of the strong convergence of $\bar{m}_{N}$ to $\bar{m}$ in $L^{2}(\bar{\Omega} \times[0, T] \times D)^{3}$, one has also :

$$
\left(\mathrm{Id}-\bar{m}_{N} \times\right) \bar{w}_{N} \underset{N \rightarrow \infty}{\rightarrow}(\mathrm{Id}-\bar{m} \times) \bar{w} \quad \text { weakly in } L^{2}(\bar{\Omega} \times[0, T] \times D)^{3} .
$$

Let us first identify the limit of $\left(\mathrm{Id}-\bar{m}_{N} \times\right) \bar{w}_{N}$.

Step 1 : let us prove that $(\operatorname{Id}-\bar{m} \times) \bar{w}(t, x) \perp \bar{m}(t, x)$ a.s.

By definition of $\bar{w}_{N}$, almost surely, and for almost every $(t, x) \in[0, T] \times D$, one has $\bar{w}_{N}(\omega, t, x) \cdot \bar{m}_{N}(\omega, t, x)=0$. Thus for any $\mathbb{R}$-valued test function $\phi \in$ $L^{\infty}(\bar{\Omega} \times[0, T] \times D)$, one has

$$
\mathbb{E}\left[\int_{0}^{T} \int_{D}\left(\bar{w}_{N} \cdot \bar{m}_{N}\right) \phi \mathrm{d} x \mathrm{~d} t\right]=0 .
$$

On the other hand, by weak convergence of $\bar{w}_{N}$ and strong convergence of $\bar{m}_{N} \phi$,

$$
\mathbb{E}\left[\int_{0}^{T} \int_{D}\left(\bar{w}_{N} \cdot \bar{m}_{N}\right) \phi \mathrm{d} x \mathrm{~d} t\right] \underset{N \rightarrow \infty}{\longrightarrow} \mathbb{E}\left[\int_{0}^{T} \int_{D}(\bar{w} \cdot \bar{m}) \phi \mathrm{d} x \mathrm{~d} t\right] .
$$


Thus, $\bar{m}(\omega, t, x) \cdot \bar{w}(\omega, t, x)=0$, for almost all $(\omega, t, x)$, and $(\operatorname{Id}-\bar{m} \times) \bar{w} \perp \bar{m}$.

Step 2 : identification of the limit for specific test functions.

We use the definition of $\bar{w}_{N}$ (55), and (10). Let us take

$$
\left.\Phi \in \mathcal{C}\left([0, T] ; L^{\infty}\left(\bar{\Omega} ; W_{x}^{1, \infty}\right)\right)\right)
$$

and consider a test function of the form

$$
\bar{m}(\omega, t, x) \times \Phi(\omega, t, x), \quad \omega \in \bar{\Omega}, t \in[0, T], x \in D .
$$

We approximate this test function by the sequence of piecewise constant functions $\left(\bar{m}_{N} \times \Phi_{N}\right)$, where we set for all $N \in \mathbb{N}^{*}$, all $0 \leq n \leq N-1$, and for $t \in[n \Delta t,(n+$ 1) $\Delta t)$,

$$
\Phi_{N}(\omega, t, x)=\Phi_{N}^{n}(\omega, x):=\Phi(\omega, n \Delta t, x)
$$

On the one hand, using the strong convergence of $\bar{m}_{N}$ to $\bar{m}$ in $L^{2}(\bar{\Omega} \times[0, T] \times D)^{3}$, and (31) we have :

$$
\begin{aligned}
& \bar{m}_{N} \times \Phi_{N} \underset{N \rightarrow \infty}{\longrightarrow} \bar{m} \times \Phi \text { strongly in } L^{2}(\bar{\Omega} \times[0, T] \times D)^{3}, \\
& \nabla\left(\bar{m}_{N} \times \Phi_{N}\right) \text { is bounded in } L^{2}(\bar{\Omega} \times[0, T] \times D)^{3 \times 3} \text { uniformly in } N,
\end{aligned}
$$

and for any $k \in\{1,2,3\}$,

$$
\bar{m}_{N} \times \partial_{x_{k}} \Phi_{N} \underset{N \rightarrow \infty}{\longrightarrow} \bar{m} \times \partial_{x_{k}} \Phi \quad \text { strongly in } L^{2}(\bar{\Omega} \times[0, T] \times D)^{3} .
$$

On the other hand, almost surely, $\left(\bar{m}_{N} \times \Phi_{N}\right) \in \overline{\mathbb{W}}_{N, n}$, and is therefore a suitable test function in the variational formulation (52). Using then (55) and the definition of $\bar{A}_{N}^{n}$, and summing on $n \in\{0, \ldots, N-1\}$, one obtains:

$$
\begin{aligned}
& \mathbb{E}\left[\int_{0}^{T}\left(\left(\mathrm{Id}-\bar{m}_{N} \times\right) \bar{w}_{N}, \bar{m}_{N} \times \Phi_{N}\right)_{2, x} \mathrm{~d} t\right] \\
& =-2 \theta \mathbb{E}\left[\int_{0}^{T}\left(\nabla \bar{v}_{N}, \nabla\left(\bar{m}_{N} \times \Phi_{N}\right)\right)_{2, x} \mathrm{~d} t\right] \\
& -2 \mathbb{E}\left[\int_{0}^{T}\left(\nabla \bar{m}_{N}, \nabla\left(\bar{m}_{N} \times \Phi_{N}\right)\right)_{2, x} \mathrm{~d} t\right] \\
& +\frac{1}{2}\left[\int_{0}^{T} \sum_{i \in \mathbb{N}}\left(\left(\operatorname{Id}-\bar{m}_{N} \times\right)\left(\left(\bar{m}_{N} \times G_{i}\right) \times G_{i}\right), \bar{m}_{N} \times \Phi_{N}\right)_{2, x} \mathrm{~d} t\right] .
\end{aligned}
$$


The first term in the right hand side above converges to zero, because of (33), and (72). For the second term, we observe that since for all $k=1,2,3$,

$$
\partial_{x_{k}} \bar{m}_{N}^{n} \cdot\left(\partial_{x_{k}} \bar{m}_{N}^{n} \times \Phi_{N}^{n}\right)=0
$$

then

$$
\begin{aligned}
2 \mathbb{E}\left[\int_{0}^{T}\left(\nabla \bar{m}_{N}, \nabla\left(\bar{m}_{N} \times \Phi_{N}\right)\right)_{2, x} \mathrm{~d} t\right] \\
=2 \mathbb{E}\left[\int_{0}^{T}\left(\sum_{k=1,2,3} \partial_{x_{k}} \bar{m}_{N}, \bar{m}_{N} \times \partial_{x_{k}} \Phi_{N}\right)_{2, x} \mathrm{~d} t\right] .
\end{aligned}
$$

By (73), and the weak convergence of $\nabla \bar{m}_{N}$ to $\nabla \bar{m}$ in $L^{2}(\bar{\Omega} \times[0, T] \times D)^{3}$ (see Remark 4), this tends to $2 \mathbb{E}\left[\int_{0}^{T} \sum_{k=1,2,3}\left(\partial_{x_{k}} \bar{m}, \bar{m} \times \partial_{x_{k}} \Phi\right)_{2, x} \mathrm{~d} t\right]$ as $N \rightarrow \infty$.

Eventually, it easily follows from assumption (8), the Sobolev embedding $H^{2}(D)^{3} \subset L^{\infty}(D)^{3}$, and the boundedness of the sequence $\left(\bar{m}_{N}\right)_{N}$ in $L^{\infty}(\bar{\Omega} \times$ $[0, T] \times D)^{3}$ and $(71)$ that the third term of the right hand side of (74) converges strongly in $L^{2}(\bar{\Omega} \times[0, T] \times D)^{3}$ to

$$
\frac{1}{2} \sum_{i \in \mathbb{N}} \mathbb{E}\left[\int_{0}^{T}\left((\mathrm{Id}-\bar{m} \times)\left(\left(\bar{m} \times G_{i}\right) \times G_{i}\right), \bar{m} \times \Phi\right)_{2, x} \mathrm{~d} t\right] .
$$

Identifying all the limits in the right hand side of (74), we get :

$$
\begin{aligned}
& \mathbb{E}\left[\int_{0}^{T}((\mathrm{Id}-\bar{m} \times) \bar{w}, \bar{m} \times \Phi)_{2, x} \mathrm{~d} t\right]=-2 \mathbb{E}\left[\int_{0}^{T}(\nabla \bar{m}, \nabla(\bar{m} \times \Phi))_{2, x} \mathrm{~d} t\right] \\
& \quad+\frac{1}{2} \sum_{i \in \mathbb{N}} \mathbb{E}\left[\int_{0}^{T}\left((\mathrm{Id}-\bar{m} \times)\left(\left(\bar{m} \times G_{i}\right) \times G_{i}\right), \bar{m} \times \Phi\right)_{2, x} \mathrm{~d} t\right]
\end{aligned}
$$

By a density argument, (75) remains true for any $\Phi \in L^{2}\left(\bar{\Omega} \times[0, T] ; H^{1}(D)\right)^{3}$.

Step 3 : identification of the limit for any test function.

We are going to use (75) with

$$
\Phi:=\bar{m} \times \Xi
$$

where $\Xi \in L^{2}\left(\bar{\Omega} \times[0, T] ; W^{1, \infty}(D)\right)^{3}$ and thus $\Phi \in L^{2}\left(\bar{\Omega} \times[0, T] ; H^{1}(D)\right)^{3}$. First, observe that for any unit vector $V \in \mathbb{S}^{2}$, one has

$$
V \times(V \times \cdot)=-\Pi_{V^{\perp}}
$$


where $\Pi_{V^{\perp}}$ denotes the orthogonal projection on $V^{\perp}$, hence from Step 1,

$$
((\operatorname{Id}-\bar{m} \times) \bar{w}) \cdot \bar{m} \times(\bar{m} \times \Xi)=-((\operatorname{Id}-\bar{m} \times) \bar{w}) \cdot \Xi
$$

Moreover, for any $1 \leq k \leq 3$, since $\bar{m} \cdot \partial_{k} \bar{m}=0$, one has

$$
\begin{aligned}
\left(\partial_{x_{k}} \bar{m} \times \bar{m}\right) \cdot \partial_{x_{k}}(\bar{m} \times \Xi) & =\left(\partial_{x_{k}} \bar{m} \times \bar{m}\right) \cdot\left(\left(\partial_{x_{k}} \bar{m} \times \Xi\right)+\left(\bar{m} \times \partial_{x_{k}} \Xi\right)\right) \\
& =\left|\partial_{x_{k}} \bar{m}\right|^{2} \bar{m} \cdot \Xi-\partial_{x_{k}} \bar{m} \cdot \partial_{x_{k}} \Xi .
\end{aligned}
$$

Using (77) and (78) in (75) with $\Phi:=\bar{m} \times \Xi$, we obtain :

$$
\begin{aligned}
- & \mathbb{E}\left[\int_{0}^{T}((\operatorname{Id}-\bar{m} \times) \bar{w}, \Xi)_{2, x} \mathrm{~d} t\right] \\
= & 2 \mathbb{E}\left[\int_{0}^{T}(\nabla \bar{m}, \nabla \Xi)_{2, x} \mathrm{~d} t\right]-2 \mathbb{E}\left[\int_{0}^{T}\left(|\nabla \bar{m}|^{2} \bar{m}, \Xi\right)_{2, x} \mathrm{~d} t\right] \\
& -\frac{1}{2} \mathbb{E}\left[\int_{0}^{T}\left(\Pi_{\bar{m}^{\perp}}\left[\sum_{i \in \mathbb{N}}(\mathrm{Id}-\bar{m} \times)\left(\left(\bar{m} \times G_{i}\right) \times G_{i}\right)\right], \Xi\right)_{2, x} \mathrm{~d} t\right],
\end{aligned}
$$

from which we deduce that

$$
(\mathrm{Id}-\bar{m} \times) \bar{w}=2\left(\Delta \bar{m}+|\nabla \bar{m}|^{2} \bar{m}\right)+\frac{1}{2} \Pi_{\bar{m}^{\perp}}\left[\sum_{i \in \mathbb{N}}(\mathrm{Id}-\bar{m} \times)\left(\left(\bar{m} \times G_{i}\right) \times G_{i}\right)\right]
$$

in $L^{2}(\bar{\Omega} \times[0, T] \times D)^{3}$.

Step 4 : end of the proof.

Note that if $V \cdot \bar{m}=0$, then

$$
(\mathrm{Id}-\bar{m} \times)^{-1} V=\frac{1}{2}(\mathrm{Id}+\bar{m} \times) V,
$$

so that

$$
\bar{w}=\Delta \bar{m}+\bar{m}|\nabla \bar{m}|^{2}+\bar{m} \times \Delta \bar{m}+\frac{1}{2} \Pi_{\bar{m} \perp} \sum_{i \in \mathbb{N}}\left(\bar{m} \times G_{i}\right) \times G_{i},
$$

and Proposition 6 is proved. 
Proof (Corollary 2) It is an immediate consequence of Proposition 6, and the fact that

$$
\mathbb{E}\left[\int_{\left[\frac{t}{\Delta t}\right] \Delta t}^{t}\left(\bar{w}_{N}(s), \Phi\right)_{2, x} \mathrm{~d} s\right] \text { tends to } 0
$$

as $N \rightarrow \infty$, for any $\Phi \in L^{2}(\bar{\Omega} \times D)^{3}$, thanks to (32).

Proposition 7 For almost every $t \in[0, T]$,

$$
\sum_{0 \leq(n+1) \Delta t \leq t}\left(\bar{m}_{N}^{n+1}-\bar{m}_{N}^{n}-\bar{v}_{N}^{n}\right)
$$

converges strongly in $L^{1}(\bar{\Omega} \times D)^{3}$ to

$$
\frac{1}{2} \int_{0}^{t} \Pi_{\bar{m}}\left[\sum_{i \in \mathbb{N}}\left(\bar{m} \times G_{i}\right) \times G_{i}\right]
$$

Corollary 3 For almost every $t \in[0, T]$,

$$
\bar{X}(t)=\bar{m}(t)-m_{0}-\int_{0}^{t}\left(\Delta \bar{m}+\bar{m}|\nabla m|^{2}+\bar{m} \times \Delta \bar{m}+\frac{1}{2} \sum_{i \in \mathbb{N}}\left(\bar{m} \times G_{i}\right) \times G_{i}\right) \mathrm{d} s,
$$

and $\bar{m} \in \mathcal{C}\left([0, T] ; L^{2}(D)^{3}\right)$.

Proof (Proposition 7.) We set for each $0 \leq n \leq N-1$ :

$$
R_{N}^{n}:=\bar{m}_{N}^{n+1}-\bar{m}_{N}^{n}-\bar{v}_{N}^{n}+\frac{1}{2}\left|\bar{A}_{N}^{n}\right|^{2} \bar{m}_{N}^{n} .
$$

It suffices to prove the following two facts :

$$
\sum_{0 \leq(n+1) \Delta t \leq t} R_{N}^{n} \underset{N \rightarrow \infty}{\longrightarrow} 0
$$

strongly in $L^{1}(\bar{\Omega} \times D)^{3}$, and

$\sum_{0 \leq(n+1) \Delta t \leq t}\left|\bar{m}_{N}^{n} \times\left(G \Delta \bar{W}_{N}^{n}\right)\right|^{2} \bar{m}_{N}^{n} \underset{N \rightarrow \infty}{\longrightarrow}-\int_{0}^{t} \Pi_{\bar{m}}\left[\sum_{i \in \mathbb{N}}\left(\bar{m} \times G_{i}\right) \times G_{i}\right] \mathrm{d} s$,

strongly in $L^{2}(\bar{\Omega} \times D)^{3}$. 


\section{Proof of (82):}

Let us decompose $R_{N}^{n}$ into four terms, namely

$$
R_{N}^{n}=\frac{\bar{m}_{N}^{n}+\bar{v}_{N}^{n}}{\sqrt{1+\left|\bar{v}_{N}^{n}\right|^{2}}}-\bar{m}_{N}^{n}-\bar{v}_{N}^{n}+\frac{1}{2}\left|\bar{A}_{N}^{n}\right|^{2} \bar{m}_{N}^{n}=I+I I+I I I+I V
$$

with

$$
\begin{gathered}
I:=\bar{m}_{N}^{n}\left(\frac{1}{\sqrt{1+\left|\bar{A}_{N}^{n}\right|^{2}}}-1+\frac{1}{2}\left|\bar{A}_{N}^{n}\right|^{2}\right), I I:=\bar{m}_{N}^{n}\left(\frac{1}{\sqrt{1+\left|\bar{v}_{N}^{n}\right|^{2}}}-\frac{1}{\sqrt{1+\left|\bar{A}_{N}^{n}\right|^{2}}}\right), \\
I I I:=\bar{v}_{N}^{n}\left(\frac{1}{\sqrt{1+\left|\bar{A}_{N}^{n}\right|^{2}}}-1\right) \text { and } I V:=\bar{v}_{N}^{n}\left(\frac{1}{\sqrt{1+\left|\bar{v}_{N}^{n}\right|^{2}}}-\frac{1}{\sqrt{1+\left|\bar{A}_{N}^{n}\right|^{2}}}\right),
\end{gathered}
$$

and treat each of them separately.

Convergence of $I$ : Using

$$
\left|\frac{1}{\sqrt{1+x^{2}}}-\left(1-\frac{1}{2} x^{2}\right)\right| \leq C x^{4}, \text { for all } x \in \mathbb{R}
$$

we get

$$
\begin{aligned}
& \mathbb{E}\left[\sum_{0 \leq(n+1) \Delta t \leq t}\left\|\bar{m}_{N}^{n}\left(\frac{1}{\sqrt{1+\left|\bar{A}_{N}^{n}\right|^{2}}}-1+\frac{1}{2}\left|\bar{A}_{N}^{n}\right|^{2}\right)\right\|_{1, x}\right] \\
& \quad \leq \frac{1}{2} \mathbb{E}\left[\sum_{0 \leq(n+1) \Delta t \leq t}\left\|\bar{A}_{N}^{n}\right\|_{4, x}^{4}\right] \\
& \leq C\|G\|_{2,1}^{4} \sum_{0 \leq(n+1) \Delta t \leq t} \Delta t^{2},
\end{aligned}
$$

which tends to zero as $N$ tends to infinity.

Convergence of $I I$ : we have by Cauchy-Schwarz inequality and a Hölder-type inequality :

$$
\begin{aligned}
& \mathbb{E}\left[\sum_{0 \leq(n+1) \Delta t \leq t}\left\|\bar{m}_{N}^{n}\left(\frac{1}{\sqrt{1+\left|\bar{v}_{N}^{n}\right|^{2}}}-\frac{1}{\sqrt{1+\left|\bar{A}_{N}^{n}\right|^{2}}}\right)\right\|_{1, x}\right] \\
& \leq C \mathbb{E}\left[\sum_{0 \leq(n+1) \Delta t \leq t}\left\|\left|\bar{v}_{N}^{n}\right|^{2}-\left|\bar{A}_{N}^{n}\right|^{2}\right\|_{1, x}\right] \\
& \quad \leq C \mathbb{E}\left[\sum_{0 \leq(n+1) \Delta t \leq t}\left\|\bar{v}_{N}^{n}+\bar{A}_{N}^{n}\right\|_{2, x}\left\|\bar{v}_{N}^{n}-\bar{A}_{N}^{n}\right\|_{2, x}\right]
\end{aligned}
$$




$$
\begin{aligned}
\leq & \frac{C}{2 \sqrt{\Delta t}} \mathbb{E}\left[\sum_{0 \leq(n+1) \Delta t \leq t}\left\|\bar{v}_{N}^{n}-\bar{A}_{N}^{n}\right\|_{2, x}^{2}\right] \\
& +\frac{C \sqrt{\Delta t}}{2} \mathbb{E}\left[\sum_{0 \leq(n+1) \Delta t \leq t}\left\|\bar{v}_{N}^{n}+\bar{A}_{N}^{n}\right\|_{2, x}^{2}\right] .
\end{aligned}
$$

We then use (15), (18) and (19) to conclude that $I I$ tends to 0 as $N$ tends to infinity. Convergence of III : Working as above, one has

$$
\begin{aligned}
& \mathbb{E}\left[\sum_{0 \leq(n+1) \Delta t \leq t}\left\|\bar{v}_{N}^{n}\left(\frac{1}{\sqrt{1+\left|\bar{A}_{N}^{n}\right|^{2}}}-1\right)\right\|_{1, x}\right] \\
& \leq \frac{\sqrt{\Delta t}}{2}\left(\sum_{0 \leq(n+1) \Delta t \leq t}\left\|\bar{v}_{N}^{n}\right\|_{2, x}^{2}\right)+\frac{C}{2 \sqrt{\Delta t}}\left(\sum_{0 \leq(n+1) \Delta t \leq t}\left\|\bar{A}_{N}^{n}\right\|_{4, x}^{4}\right) .
\end{aligned}
$$

Then, using again (19), and (16), the above quantity tends to 0 as $N \rightarrow \infty$.

Convergence of $I V$ : Cauchy-Schwarz inequality implies :

$$
\begin{aligned}
& \mathbb{E}\left[\left\|\bar{v}_{N}^{n}\left(\frac{1}{\sqrt{1+\left|\bar{v}_{N}^{n}\right|^{2}}}-\frac{1}{\sqrt{1+\left|\bar{A}_{N}^{n}\right|^{2}}}\right)\right\|_{1, x}\right] \\
& \leq\left(\mathbb{E}\left[\sum_{0 \leq(n+1) \Delta t \leq t}\left\|\bar{v}_{N}^{n}\right\|_{2, x}^{2}\right]\right)^{\frac{1}{2}}\left(\mathbb{E}\left[\sum_{0 \leq(n+1) \Delta t \leq t}\left\|\bar{v}_{N}^{n}-\bar{A}_{N}^{n}\right\|_{2, x}^{2}\right]\right)^{\frac{1}{2}} .
\end{aligned}
$$

Using (18) and (19), we conclude that $I V$ tends to 0 as $N \rightarrow \infty$. Finally (82) is proved.

Proof of (83).

For each $N \in \mathbb{N}^{*}$, and $0 \leq n \leq N$, observe that if we denote by $\mathbb{E}\left[\cdot \mid \overline{\mathbb{F}}_{N}^{n}\right]$ the conditional expectation with respect to $\overline{\mathbb{F}}_{N}^{n}$ (see (59)), we have

$$
\mathbb{E}\left[\left|\bar{m}_{N}^{n} \times\left(G \Delta \bar{W}_{N}^{n}\right)\right|^{2} \mid \mathbb{F}_{N}^{n}\right]=\Delta t \sum_{i \in \mathbb{N}}\left|\bar{m}_{N}^{n} \times G_{i}\right|^{2}
$$

We set

$$
\sum_{0 \leq(n+1) \Delta t \leq t} \bar{m}_{N}^{n}\left|\bar{m}_{N}^{n} \times\left(G \Delta \bar{W}_{N}^{n}\right)\right|^{2}-\int_{0}^{t} \sum_{i \in \mathbb{N}} \bar{m}\left|\bar{m} \times G_{i}\right|^{2} \mathrm{~d} s=I+I I
$$

where

$$
I=\sum_{0 \leq(n+1) \Delta t \leq t} \bar{m}_{N}^{n}\left(\left|\bar{m}_{N}^{n} \times\left(G \Delta \bar{W}_{N}^{n}\right)\right|^{2}-\sum_{i \in \mathbb{N}}\left|\bar{m}_{N}^{n} \times G_{i}\right|^{2} \Delta t\right),
$$


and

$$
I I=\sum_{0 \leq(n+1)} \sum_{\Delta t \leq t} \bar{m}_{N}^{n}\left|\bar{m}_{N}^{n} \times G_{i}\right|^{2} \Delta t-\int_{0}^{t} \sum_{i \in \mathbb{N}} \bar{m}\left|\bar{m} \times G_{i}\right|^{2} \mathrm{~d} s .
$$

Let us prove that the first term above tends to zero as $N$ tends to infinity in $L^{2}(\bar{\Omega} \times$ $D)^{3}$.

$$
\mathbb{E}\left[\|I\|_{2, x}^{2}\right]=\mathbb{E}\left[\left\|\sum_{0 \leq(n+1) \Delta t \leq t} \bar{m}_{N}^{n}\left(\left|\bar{m}_{N}^{n} \times\left(G \Delta \bar{W}_{N}^{n}\right)\right|^{2}-\sum_{i \in \mathbb{N}}\left|\bar{m}_{N}^{n} \times G_{i}\right|^{2} \Delta t\right)\right\|_{2, x}^{2}\right]
$$

Developing the square under the expectation above, we get a sum over two indices $k \leq n$, which contains the following terms :

$$
\begin{gathered}
\mathbb{E}\left[\left(\bar{m}_{N}^{n}\left(\left|\bar{m}_{N}^{n} \times\left(G \Delta \bar{W}_{N}^{n}\right)\right|^{2}-\sum_{i \in \mathbb{N}}\left|\bar{m}_{N}^{n} \times G_{i}\right|^{2} \Delta t\right),\right.\right. \\
\left.\left.\bar{m}_{N}^{k}\left(\left|\bar{m}_{N}^{k} \times G \Delta \bar{W}_{N}^{k}\right|^{2}-\sum_{i \in \mathbb{N}}\left|\bar{m}_{N}^{k} \times G_{i}\right|^{2} \Delta t\right)\right)_{2, x}\right] .
\end{gathered}
$$

When $k<n$, this is equal to

$$
\begin{gathered}
\mathbb{E}\left[\left(\bar{m}_{N}^{n} \mathbb{E}\left[\left|\bar{m}_{N}^{n} \times\left(G \Delta \bar{W}_{N}^{n}\right)\right|^{2}-\sum_{i \in \mathbb{N}}\left|\bar{m}_{N}^{n} \times G_{i}\right|^{2} \Delta t \mid \overline{\mathbb{F}}_{N}^{n}\right],\right.\right. \\
\left.\left.\bar{m}_{N}^{k}\left(\left|\bar{m}_{N}^{k} \times G \Delta \bar{W}_{N}^{k}\right|^{2}-\sum_{i \in \mathbb{N}}\left|\bar{m}_{N}^{k} \times G_{i}\right|^{2} \Delta t\right)\right)_{2, x}\right] .
\end{gathered}
$$

Thus, using (84), these terms vanish. It follows, using again (84)

$$
\begin{aligned}
\mathbb{E}\left[\|I\|_{2, x}^{2}\right] & =\sum_{0 \leq(n+1) \Delta t \leq t} \mathbb{E}\left[\left\|\left|\bar{m}_{N}^{n} \times\left(G \Delta \bar{W}_{N}^{n}\right)\right|^{2}-\sum_{i \in \mathbb{N}}\left|\bar{m}_{N}^{n} \times G_{i}\right|^{2} \Delta t\right\|_{2, x}^{2}\right] \\
& =\sum_{0 \leq(n+1) \Delta t \leq t} \mathbb{E}\left[\int_{D}\left|\bar{m}_{N}^{n} \times\left(G \Delta \bar{W}_{N}^{n}\right)\right|^{4}-\left(\sum_{i \in \mathbb{N}}\left|\bar{m}_{N}^{n} \times G_{i}\right|^{2}\right)^{2} \Delta t^{2} \mathrm{~d} x\right] .
\end{aligned}
$$

Both terms on the right hand side are bounded by $C(T)\|G\|_{2,1}^{4} \Delta t$ [see (16)], hence tend to zero as $N$ tends to infinity.

For $I I$, we write

$$
\sum_{0 \leq(n+1) \Delta t \leq t} \sum_{i \in \mathbb{N}} \bar{m}_{N}^{n}\left|\bar{m}_{N}^{n} \times G_{i}\right|^{2} \Delta t=\int_{0}^{\left[\frac{t}{\Delta t}\right] \Delta t} \sum_{i \in \mathbb{N}} \bar{m}_{N}\left|\bar{m}_{N} \times G_{i}\right|^{2} \mathrm{~d} s,
$$


and note that $\int_{\left[\frac{t}{\Delta t}\right] \Delta t}^{t} \sum_{i \in \mathbb{N}} \bar{m}_{N}\left|\bar{m}_{N} \times G_{i}\right|^{2} \mathrm{~d} s$ tends to zero in $L^{2}(\bar{\Omega} \times D)^{3}$. Moreover, we have that

$$
\begin{aligned}
& \mathbb{E}\left[\left\|\int_{0}^{t} \sum_{i \in \mathbb{N}} \bar{m}_{N}\left|\bar{m}_{N} \times G_{i}\right|^{2} \mathrm{~d} s-\int_{0}^{t} \sum_{i \in \mathbb{N}} \bar{m}\left|\bar{m} \times G_{i}\right|^{2} \mathrm{~d} s\right\|_{2, x}\right] \\
& \leq C\|G\|_{2,2}^{2} \mathbb{E} \int_{0}^{T}\left\|\bar{m}_{N}-\bar{m}\right\|_{2, x} \mathrm{~d} s
\end{aligned}
$$

tends to 0 as $N$ tends to infinity. We conclude that

$$
\sum_{0 \leq(n+1) \Delta t \leq t} \sum_{i \in \mathbb{N}} \bar{m}_{N}^{n}\left|\bar{m}_{N}^{n} \times G_{i}\right|^{2} \Delta t \underset{N \rightarrow \infty}{\longrightarrow} \int_{0}^{t} \sum_{i \in \mathbb{N}} \bar{m}\left|\bar{m} \times G_{i}\right|^{2} \mathrm{~d} s
$$

strongly in $L^{1}\left(\bar{\Omega} ; L^{2}(D)^{3}\right)$. Eventually, (83) follows from

$$
\int_{0}^{t} \sum_{i \in \mathbb{N}} \bar{m}\left|\bar{m} \times G_{i}\right|^{2} \mathrm{~d} s=-\int_{0}^{t} \Pi_{\bar{m}} \sum_{i \in \mathbb{N}}\left(\left(\bar{m} \times G_{i}\right) \times G_{i}\right) \mathrm{d} s .
$$

Proof (Corollary 3.) We recall that we have for almost every $t \in[0, T]$ and for all $N \in \mathbb{N}^{*}$

$$
\bar{X}_{N}(t)=\bar{m}_{N}(t)-m_{0}-\sum_{0 \leq(n+1) \Delta t \leq t} \Delta t \bar{w}_{N}^{n}-\sum_{0 \leq(n+1) \Delta t \leq t}\left(\bar{m}_{N}^{n+1}-\bar{m}_{N}^{n}-\bar{v}_{N}^{n}\right)
$$

and that, up to the extraction of a subsequence,

$$
\bar{m}_{N} \longrightarrow \bar{m} \quad \text { strongly in } L^{2}(\bar{\Omega} \times[0, T] \times D)^{3},
$$

and thus without loss of generality, we can assume that for almost any $t \in[0, T]$,

$$
\bar{m}_{N}(t) \longrightarrow \bar{m}(t) \quad L^{2}(\bar{\Omega} \times D)^{3} .
$$

Then, thanks to Corollary (2) and Proposition 7,

$$
-\sum_{0 \leq(n+1) \Delta t \leq t} \Delta t \bar{w}_{N}^{n}-\sum_{0 \leq(n+1) \Delta t \leq t}\left(\bar{m}_{N}^{n+1}-\bar{m}_{N}^{n}-\bar{v}_{N}^{n}\right)
$$


converges weakly in $L^{2}(\bar{\Omega} \times D)^{3}$ to

$$
\begin{aligned}
& -\int_{0}^{t}\left(\Delta \bar{m}+\bar{m}|\nabla \bar{m}|^{2}+\bar{m} \times \Delta \bar{m}+\frac{1}{2} \Pi_{\bar{m} \perp}\left[\sum_{i \in \mathbb{N}}\left(\bar{m} \times G_{i}\right) \times G_{i}\right]\right) \mathrm{d} s \\
& -\frac{1}{2} \int_{0}^{t}\left(\Pi_{\bar{m}}\left[\sum_{i \in \mathbb{N}}\left(\bar{m} \times G_{i}\right) \times G_{i}\right]\right) \mathrm{d} s
\end{aligned}
$$

The continuity of $\bar{m}$ follows from (80), the continuity of $\bar{X}$ (Proposition 4 ), and the fact that $\bar{w} \in L^{1}\left(0, T ; L^{2}(D)^{3}\right)$ a.s. (see Proposition 6 ).

End of proof of Theorem 1. The conclusion follows, thanks to (80), together with (67): there exists a martingale solution $\left(\tilde{\Omega}, \tilde{\mathbb{P}}, \tilde{\mathcal{F}}_{t \in[0, T]}, \tilde{W}, \bar{m}\right)$ of $(5)$, i.e. for any time $t \in[0, T]$,

$$
\begin{aligned}
\bar{m}(t)= & m_{0}+\int_{0}^{t}\left(\Delta \bar{m}+\bar{m}|\nabla \bar{m}|^{2}+\bar{m} \times \Delta m+\frac{1}{2} \sum_{i \in \mathbb{N}}\left(\bar{m} \times G_{i}\right) \times G_{i}\right) \mathrm{d} s \\
& +\int_{0}^{t} \bar{m} \times(G \mathrm{~d} \tilde{W}(s)) .
\end{aligned}
$$

Acknowledgments Partial funding of this research through the ANR projects Micro-MANIP (ANR-08BLAN-0199) and STOSYMAP (ANR-2011-BS01-015-03) is gratefully acknowledged.

\section{References}

1. Brown, W.F.: Thermal fluctuations of a single-domain particle. Phys. Rev. 130(5), 1677 (1963)

2. Landau, L.D., Lifshitz, E.: On the theory of the dispersion of magnetic permeability in ferromagnetic bodies. Phys. Z. Sowjetunion 8(153), 101-114 (1935)

3. Brown, W.F.: Micromagnetics. Interscience, New York (1963)

4. Alouges, F., Soyeur, A.: On global weak solutions for Landau-Lifshitz equations: existence and nonuniqueness. SIAM J. Numer. Anal. 44(4), 1405-1419 (2006)

5. Carbou, G., Fabrie, P.: Comportement asymptotique des solutions faibles des équations de LandauLifschitz. Comptes Rendus de l'Académie des Sciences-Series I-Mathematics 325(7), 717-720 (1997)

6. Carbou, G., Fabrie, P.: Regular solutions for Landau-Lifschitz equation in a bounded domain. Differ. Integral Equ. 14(2), 213-229 (2001)

7. Carbou, G., Fabrie, P.: Regular solutions for Landau-Lifschitz equation in $\mathbb{R}^{3}$. Commun. Appl. Anal. 5(1), 17-30 (2001)

8. d'Aquino, M., Serpico, C., Coppola, G., Mayergoyz, I., Bertotti, G.: Midpoint numerical technique for stochastic Landau-Lifshitz-Gilbert dynamics. J. Appl. Phys. 99(8), 08B905-08B905 (2006)

9. Serpico, C., Mayergoyz, I., Bertotti, G.: Numerical technique for integration of the Landau-Lifshitz equation. J. Appl. Phys. 89(11), 6991-6993 (2001)

10. Serpico, C., Mayergoyz, I., Bertotti, G.: Analytical solutions of Landau-Lifshitz equation for precessional switching. J. Appl. Phys. 93(10), 6909-6911 (2003)

11. Visintin, A.: On Landau-Lifshitz equations for ferromagnetism. Jpn. J. Appl. Math. 2(1), 69-84 (1985)

12. Banas, L., Brzeźniak, Z., Neklyudov, M., Prohl, A.: Stochastic ferromagnetism-analysis and numerics. De Gruyter (2013) 
13. Banas, L., Brzeźniak, Z., Neklyudov, M., Prohl, A.: A convergent finite-element-based discretization of the stochastic Landau-Lifshitz-Gilbert equation. J. Numer. Anal. 34(2), 502-549 (2013)

14. Banas, L., Brzeźniak, Z., Prohl, A.: Computational studies for the stochastic Landau-Lifshitz-Gilbert equation. SIAM J. Sci. Comput. 35(1), B62-B81 (2013)

15. Brzeźniak, Z., Goldys, B., Jegaraj, T.: Weak solutions of a stochastic Landau-Lifshitz-Gilbert equation. Appl. Math. Res. Express AMRX 1, 1-33 (2013)

16. Berkov, D.: Magnetization dynamics including thermal fluctuations. In: Kronmüller, H., Parkin, S. (eds.) Handbook of Magnetism and Advanced Magnetic Materials, vol. 2. Wiley, Chichester (2007)

17. Van Kampen, N.: Itô versus Stratonovich. J. Stat. Phys. 24(1), 175-187 (1981)

18. Reznikoff, M.G.: Rare Events in Finite and Infinite Dimensions. Ph.D. thesis, New York University (2004)

19. Cimrák, I.: Error estimates for a semi-implicit numerical scheme solving the Landau-Lifshitz equation with an exchange field. IMA J. Numer. Anal. 25(3), 611-634 (2005)

20. Cimrák, I.: A survey on the numerics and computations for the Landau-Lifshitz equation of micromagnetism. Arch. Comput. Methods Eng. 15(3), 1-37 (2007)

21. Cimrák, I., Slodička, M.: An iterative approximation scheme for the Landau-Lifshitz-Gilbert equation. J. Comput. Appl. Math. 169(1), 17-32 (2004)

22. Alouges, F.: A new finite element scheme for Landau-Lifschitz equations. Discret. Contin. Dyn. Syst. Ser. 1(2), 187-196 (2008)

23. Alouges, F., Jaisson, P.: Convergence of a finite element discretization for the Landau-Lifshitz equations in micromagnetism. Math. Models Methods Appl. Sci. 16(02), 299-316 (2006)

24. Bartels, S., Ko, J., Prohl, A.: Numerical analysis of an explicit approximation scheme for the LandauLifshitz-Gilbert equation. Math. Comput. 77(262), 773-788 (2008)

25. Kritsikis, E., Vaysset, A., Buda-Prejbeanu, L., Alouges, F., Toussaint, J.C.: Beyond first-order finite element schemes in micromagnetics. J. Comput. Phys. 256, 357-366 (2014). doi:10.1016/j.jcp.2013. 08.035

26. Goldys, B., Le, K.N., Tran, T.: A finite element approximation for the stochastic Landau-LifshitzGilbert equation (2013)

27. Doss, H.: Liens entre équations différentielles stochastiques et ordinaires. Ann. Inst. H. Poincaré Sect. B (N.S.) 13(2), 99-125 (1977)

28. Sussmann, H.J.: An interpretation of stochastic differential equations as ordinary differential equations which depend on the sample point. Bull. Am. Math. Soc. 83(2), 296-298 (1977)

29. Lelièvre, T., Le Bris, C., Vanden-Eijnden, E.: Analyse de certains schémas de discrétisation pour des équations différentielles stochastiques contraintes. Comptes Rendus Mathematique 346(7), 471-476 (2008)

30. Adams, R.A., Fournier, J.J.F.: Sobolev Spaces, Pure and Applied Mathematics (Amsterdam), vol. 140, 2nd edn. Elsevier/Academic Press, Amsterdam (2003)

31. Alouges, F.: A new algorithm for computing liquid crystal stable configurations: the harmonic mapping case. SIAM J. Numer. Anal. 34(5), 1708-1726 (1997)

32. Da Prato, G., Zabczyk, J.: Stochastic Equations in Infinite Dimensions. Cambridge University Press, Cambridge (2008)

33. Flandoli, F., Gatarek, D.: Martingale and stationary solutions for stochastic Navier-Stokes equations. Probab. Theory Relat. Fields 102(3), 367-391 (1995)

34. Assouad, P.: Espaces $p$-lisses et $q$-convexes. inégalités de Bürkholder. Séminaire d'Analyse fonctionnelle pp. 1-7 (1975) 